\title{
Evaluating the Effectiveness of the Lateral Intercrural Suture to Decrease the Interdomal Distance to Improve the Definition of the Nasal Tip in Primary Rhinoplasty
}

\author{
Caio Márcio Correia Soares ${ }^{1}$ Marcos Mocelin ${ }^{2}$ Rogerio Pasinato ${ }^{2}$ Cezar Augusto Sarraf Berger ${ }^{1}$ \\ Francisco Luiz Busato Grocoske ${ }^{3}$ Maria Julia Abrão Issa ${ }^{2}$
}

${ }^{1}$ Department of Otolaryngology, Universidade Federal do Paraná (UFPR), Curitiba/PR, Brazil

2 Department of Otorhinolaryngology, Universidade Federal do Paraná Address for correspondence Caio Márcio Correia Soares, MS, Av. (UFPR), Curitiba/PR, Brazil

3 Physician Fellowship Program 2012, Hospital IPO, Curitiba/PR, Brazil Republica Argentina 2069, Curitiba/PR 80620-010, Brazil (e-mail: soares_caio@hotmail.com).

Int Arch Otorhinolaryngol 2014;18:92-107.

\begin{abstract}
Introduction Several surgical techniques emphasizing sutures on the lower lateral cartilage have been studied by surgeons as instruments to improve nasal tip remodeling. It is already known that the domal divergence angle and its definition angle can be modified by lateral intercrural suture (LIS). Techniques for measuring these structures are not yet standardized.

Objectives Assess the efficacy of LIS using polydioxanone 4-0 absorbable thread by interdomal distance and systematize the LIS technique to improve nasal tip definition. Materials and Methods This prospective study measured and analyzed interdomal distances measured preoperatively and perioperatively compared with 3-and 6-month postoperative measurements.

Keywords

- rhinoplasty

- polydioxanone

- suture techniques

Results LIS was efficient on reducing interdomal distances.

Conclusion LIS is statistically safe and efficient and has low morbidity when utilized in patients with mild to moderate deformities, because it reduces the domal divergence angle, effectively sustaining the nasal tip.
\end{abstract}

\section{Introduction}

Rhinoplasty is one of the most common surgeries in our field, and its demand requires wider studies aiming at offering the patient more durable, consistent, predictable, and harmonic results. The nose, the focus of several studies, must also present an aesthetic balance between dynamic and functional, especially because the nasal tip represents the main opportunity for postoperative dissatisfaction. ${ }^{1}$

In addition to aesthetics analysis, the surgeon must evaluate the respiratory function to provide an aesthetically bal- anced and functionally efficient nose. ${ }^{2}$ The treatment of the nasal tip is one of the most important components on rhinoplasty, because modifying its form involves mainly the control, distribution, and proportion of lower lateral cartilages (LLCs). The utilization of sutures on specific areas of LLCs, as well as on adjacent soft tissues, is particularly useful in rhinoplasty ${ }^{3}$; therefore, permanent or semipermanent sutures are necessary to maintain the cartilage on the intended position until fibrosis, resulting from time and cicatrization, is well underway. All sutures that remain more than 6 months will probably be satisfactory, because the scar will received

January 8, 2013

accepted

June 6, 2013
DOI http://dx.doi.org/

10.1055/s-0033-1352505. ISSN 1809-9777.
Copyright $\odot 2014$ by Thieme Publicações License terms Ltda, Rio de Janeiro, Brazil

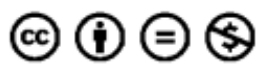


provide enough support of the LLC after absorption of its thread. ${ }^{4}$ This concept of suture is based on otoplasty techniques for the correction of floppy ears.

On the other hand, these sutures maintain and improve the structural support mechanism of the tip, considering that this area is physiologically dynamic, moving during inhalation and exhalation and contributing to facial expression. It works as a damper during nasal trauma and is an icon of nasal beauty and consequently facial beauty. ${ }^{5}$

O'Neal et al have described the domal definition angle and the domal divergence angle. ${ }^{6}$ This anatomic concept allows the surgeon to correlate the proportion and form of the nasal tip with sutures that modify those angles, offering better definition of the nasal tip.

The domal angle is demarcated by the lateral crus, and the medial or intermediate crus, when there is one. An interdomal divergence angle of 30 degrees is considered normal, and the domal arch, $\leq 4 \mathrm{~mm}^{7}$

For harmonious aesthetic lines on the nasal dorsum, slightly divergent curves must occur, starting on the superciliary arch and extending to the nasal tip definition points that correspond to the domes. A poorly defined nasal tip, such as in bulbous or boxy noses, have an angle wider than 30 degrees. When observed through a basal view, it gains a square shape, and its correction includes repositioning of nasal tip definition points, domes angling, and modeling of lateral crura, aiming for a more triangular form from a basal view.

\section{Objectives}

1. Assess the efficacy of the lateral intercrural suture (LIS) using polydioxanone (PDS) 4-0 absorbable thread by the interdomal measure on primary rhinoplasty.

2. Systematize and describe the LIS to improve nasal tip definition on primary rhinoplasty with mild to moderate deformities.

\section{Literature Review}

Detailed knowledge of the nasal anatomy is the most important element for the perioperative identification of anatomic structures and is directly related to the choice of the surgical technique that will be utilized. The nasal tip has a variable complex structure, with anatomic structures of several shapes, consistency, and volumes, intimately connected. In other words, it is composed of structures of different characteristics that function as a whole, and any alteration in one of theme may affect another, with different aesthetic-functional results.

The nasal lobe is an area that includes the nasal tip and is demarcated by a line connecting the upper edge of the nostrils, the supratip (breakpoint), and the anterior half of the lateral nasal wall. It is divided in tip, supratip, and infratip.

The LLC is the main cartilage of the nasal tip and is anatomically divided into the medial, intermediate, and lateral crus. From the lateral border of the lateral crus to the pyriform aperture, we have the sesamoid or auxiliary cartilages that are bound by a fibrofatty connective tissue (-Figs. 1 and 2$)^{8}$

The LLC is a complex and variable structure. Traditionally, were only considered the medial and lateral crura connected by the domal segment. However, Sheen and Sheen introduced the concept of intermediate crus on which the domal segment would be superior. ${ }^{9}$ There are still some controversies about considering the intermediate crus as a single structure, but surgically there are few doubts regarding its importance on the nasal lobe configuration.

The medial crus of the LLC consists of the base and columellar segments. Oneal and Beil made clear that the intermediate crus is more than just a connection between the medial and lateral crura. ${ }^{10}$ It has a complex structure and therefore is important for the nasal lobe.

Most of the patients present an angulation in two planes: the cephalic rotation angle and the basal divergence angle (-Fig. 3).

These angles affect the shape and the protuberance of the large part of the columella. The amount of soft tissue, as well as the caudal portion of the septum, interferes on the projection of the nasal tip. Anatomically the columellar segment begins on the upper limit of the basal segment and ends on the columellar breakpoint, where the intermediate crus of the LLC starts. Variations on the width of the columellar supratip occur frequently. Laterally, the more convex portion of the columella corresponds to the same columellar breakpoint and to the junction of this segment with the intermediate crus. The more acute the angulation, the more elongated the columella will be (-Fig. 4).

The intermediate crus combine the lobular and domal segment, or simply the dome. The lobular segment has a

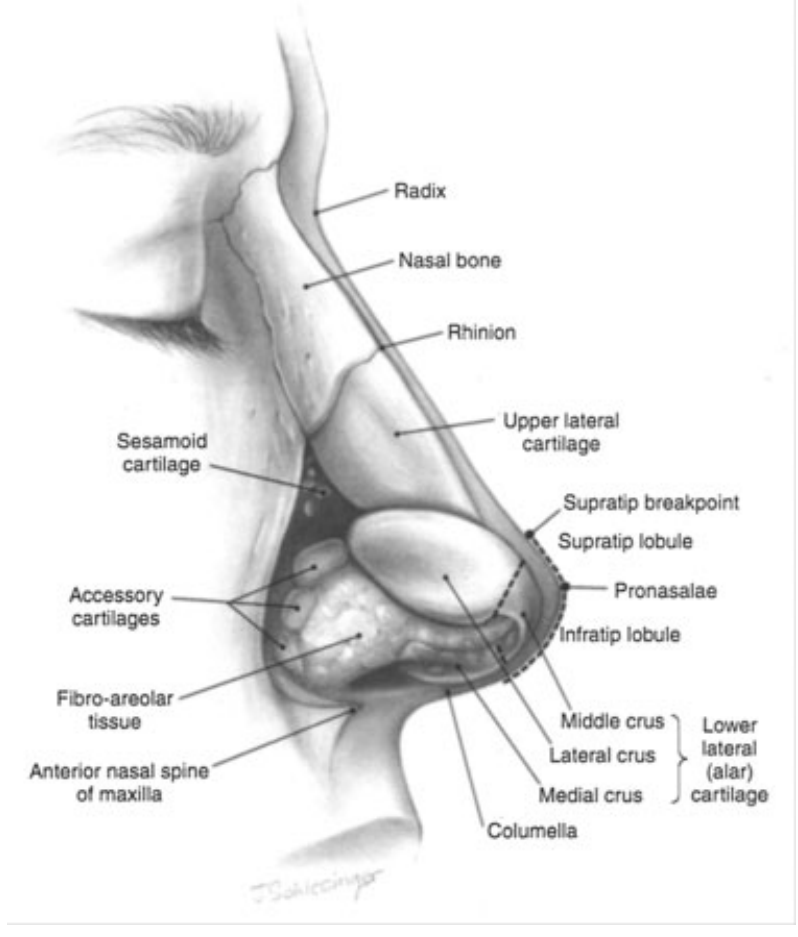

Fig. 1 Right lateral view of the nose. Source: Oneal et al. ${ }^{39}$ 


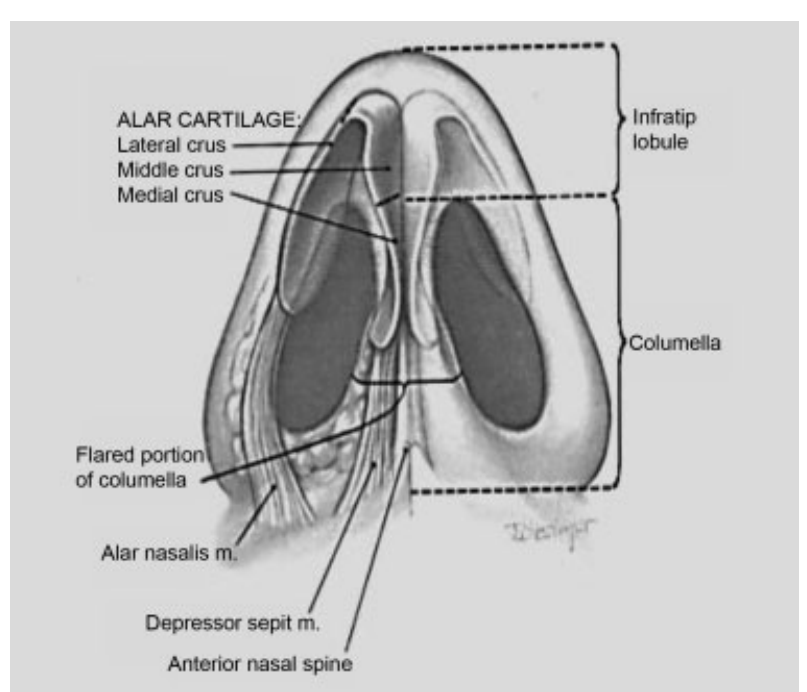

Fig. 2 View from the nasal base. Source: Oneal et al. ${ }^{39}$

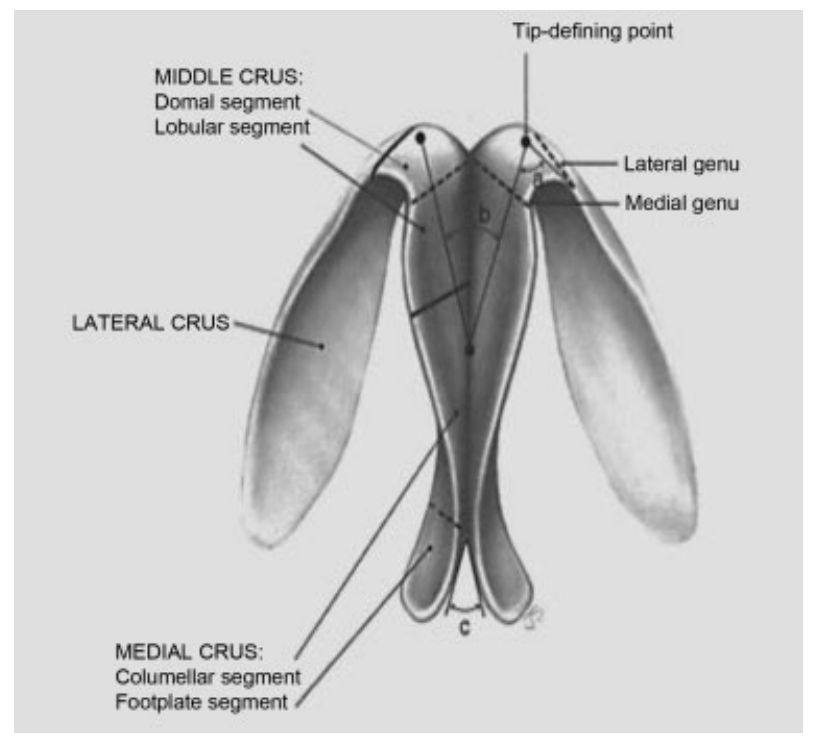

Fig. 3 Basal view of the nose presenting the domal divergence and domal definition angles: (a) domal definition angle, (b) domal divergence angle, (c) base divergence angle. Source: Oneal et al. ${ }^{39}$

more variable structure; however, it exerts less influence over the external appearance because normally it is covered by a great amount of soft tissue. Its width and angle determine the shape and protrusion of the infratip.

The domal segment, on the other hand, stays more visible because it is covered only by a small portion of soft tissue and by thin skin. Daniel describes three curvatures on the domal region: (1) convex, where the domes form a gentle triangle that is important to the harmony of the nostril apex, where cartilage and skin have direct contact; (2) boxy nose, characterizing poor definition of the nasal tip; (3) concave, forming a double dome, which is less frequent. ${ }^{11}$

The lateral crus is the larger component of the nasal tip and shapes the upper and anterior portions of the alar wall. Medially it is continuous to the domal segment of the intermediate crus and laterally to the accessory cartilages

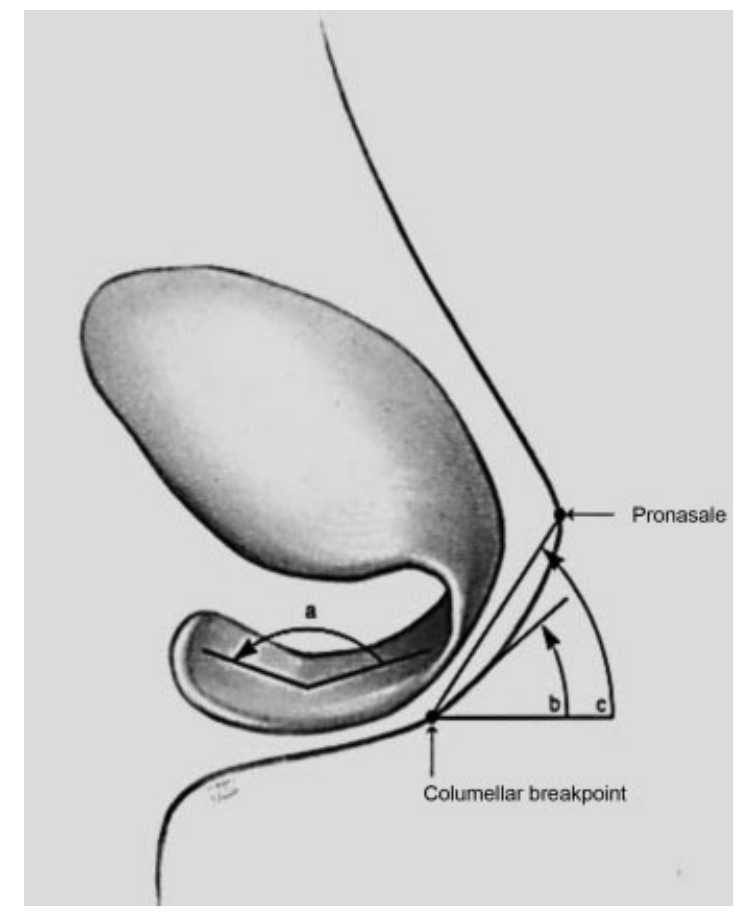

Fig. 4 Lateral view of the lateral cartilage: (a) cephalic rotation angle, (b) columellar angle, (c) angle of tip rotation. Source: Oneal et al. ${ }^{39}$

on the pyriform process region. Its caudal border offers support and definition to the anterior alar edge. Laterally it curves in a cephalic way, becoming wider. Surgically the approximation between the lateral crura increases the projection of the nasal tip and simultaneously gives it a better definition, distinguishing the supratip. Zelnik and Gingrass mentioned that when the portion adjacent to the dome is concave, this promotes its definition, requiring a minimum modification of the lateral crus. ${ }^{12}$ However, it is known that the nasal tip definition is a subjective characteristic for which there is no exact formula to obtain a standard shape.

The scroll region is the junction of the cephalic border and the lateral crus of the LLC. Usually the caudal border of the upper lateral cartilage has a slight lateral curve and the LLC has a curve toward the bottom, lifting the LLC over the upper lateral crus. The wider the curve of this area, the greater the bulbosity of the nasal lobe will be, resulting in poor definition.

Daniel affirms that to improve the nasal tip definition we should convert the convex lateral crus into a concave form, and by this way highlighting the dome definition, where the tips, aesthetically adjusted, would give the characteristics of a convex domal segment and a concave lateral crus. ${ }^{11}$

Sheen and Sheen analyzed the ideal nasal tip shape and described it as two equilateral triangles with a common base made by the line that unites the domes. Therefore, they named it the intercrural distance, the distance between the two domes that coincide on the common base of the triangles. ${ }^{9}$

Toriumi and Tardy assessed the asymmetry and concavities using tridimensional figures, to document precisely the spot of imperfections that are emphasized by shadows that 
impact nasal aesthetic contour. ${ }^{13}$ From a frontal view, there are criteria for a subtle transition from the lobe to the wing. The authors conclude that tip definition points are horizontally enhanced with tenuous shadows above and beneath, with two opposite horizontal curvy lines outlining the tip enhancement. The exact configuration of this enhancement varies among each patient, but on most females, the width is $8 \mathrm{~mm}$, varying from 6 to $14 \mathrm{~mm}$. These values are a little larger on men (-Fig. $\mathbf{5}$ ).

On oblique view, there is a light shadow on the supratip break that continues along the supra alar notch. When we decide on a cephalic resection of the LLC, we cause the break in the supratip that descends on a frontal view resulting in a better definition of the nasal tip. The contour of the infratip break also becomes important to improve natural aesthetic aspects.

From a basal view, the cartilage arch must have a triangular shape free from pinching. The soft tissue triangles must be bland. On a lateral view, the nasal tip must be a little above the dorsum with a slight break of the supratip. These shadows are intensified on the soft tissue triangle. This leads us to conclude that to be well defined, a nasal tip should have a supratip and an infratip break, with the supratip defined by the junction of the lobe and the nasal dorsum and the infratip by the junction of the lobe with the columella. On the transition of these regions, there must be a gap highlighting the lobe from the tip and nasal dorsum.

Yet it is worth noting that the nasal dorsum aesthetic line, from the eyebrow to the tip, must be soft and its surface free from roughness (-Fig. 6 ).

There is a firm transversal connective tissue that unites the medial and intermediate crura. Previously, it formed the interdomal ligament found cephalically with vertical and longitudinal fibers of the overlying dermis forming what Pitanguy described as dermocartilaginous ligament of the nose. ${ }^{14}$ This ligament would make the superficial connection, helping the dynamic balance between the dorsum and the tip of the nose. Therefore its section would result on a cephalic release of the nasal tip, especially in round noses.

According to Tebbetts, a strong unification of the medial and intermediate crura by the fibrous tissue would provide a
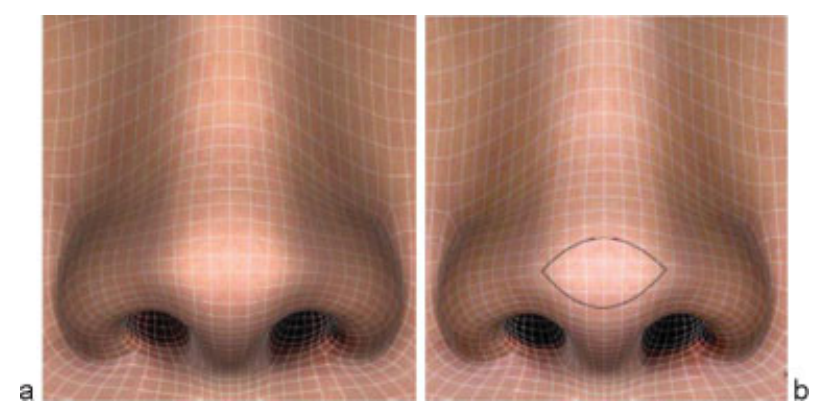

Fig. 5 Analysis performed by Toriumi ${ }^{13}$ presenting what would be a favorable contour from an anterior view. (a) Subtle transition from the lobe to the wing. (b) Two opposite horizontal curvy lines outlining the nasal tip enhancement. Source: Toriumi. ${ }^{13}$

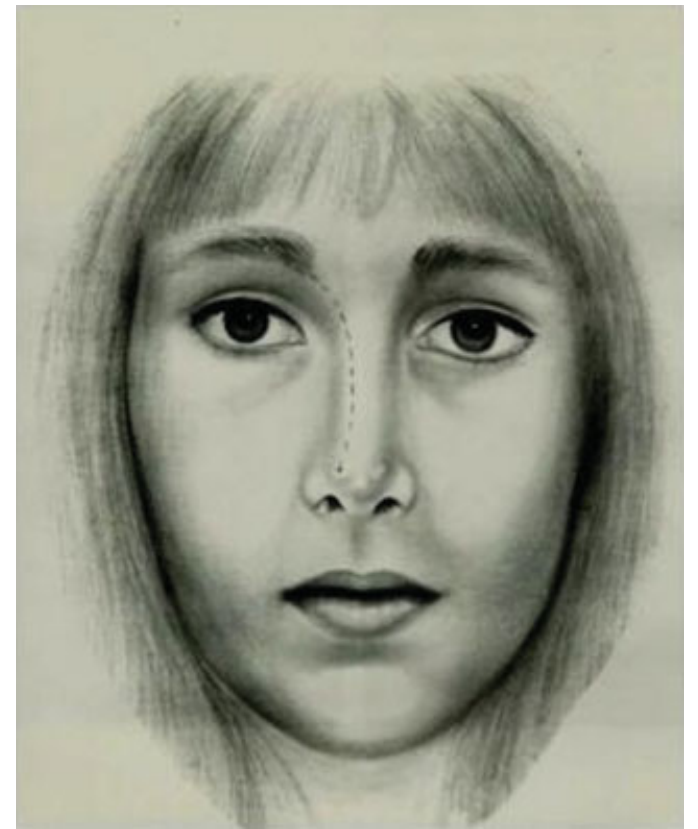

Fig. 6 Aesthetic line of the nasal dorsum. Source: Sheen and Sheen. ${ }^{40}$

unique functional structure, composed by the lateral crura of the LLC, a sustenance tripod to the nasal tip. ${ }^{15}$

The thickness of skin requires special attention on a rhinoplasty surgical plan. Usually the skin tends to be thinner on the upper half of the nose and thicker and more adherent on the posterior half. Thicker skin disguises greater defects, but also soothes surgical corrections. Oiliness produced by the sebaceous glands on the nose tip make its definition difficult, mainly on ethnics or non-Caucasian noses. On elderly patients, modification of cutaneous characteristics can also be responsible for some alteration typically related to aging, such as nasal tip drop or lengthened nose.

\section{Materials and Methods}

The present study was approved by the ethics committee under the number CAE 0182.0208.000-11.

This is a prospective experimental divided into the following steps: selection of the target population, clinical management of rhinoplasty, collection of photographic evidence and measurement of the interdomal distance, evaluation and interpretation of data, and statistical analysis.

The methodology used in this study development can be divided didactically into five phases:

1. Creation of a theoretical basis for clinical data collection regarding otolaryngology

2. Use of computer program for computerization of the database

3. Incorporation of the protocol to the Integrated Electronic Protocols

4. Application of computerized protocol, in the form of a pilot project 
5. Analysis of the results obtained by the data collection pilot project

\section{Sample Characterization and Research Site}

The present experimental prospective study was performed between March 2010 and November 15, 2011. In addition to theoretical research, the present study was composed of four basic protocols:

- Selection of target population according to criteria of rhinoplasty indications associated or not with septoplasty

- Clinical control of the interdomal distance in preoperative, perioperative, and postoperative periods, with control and measurement at 90 to 180 days, respectively

- Photographic control and measurement of interdomal distance

- Control, assessment, and interpretation of the collected data and statistical results

At this step, the electronic protocol SINPE (Sistema Integrado de Protocolos Eletrônicos) was followed to collect and hierarchize the research steps that corresponded to anamnesis, physical exams, complementary exams, diagnosis, and surgical treatment.

The average interdomal distances were compared during different operative stages from the whole group, between genders, and between skin types of the target population utilizing statistical Mann-Whitney test. The adopted significance level was $p \leq 0.05$.

\section{Selection of Patients}

Patients were 17 to 62 years old, of both sexes, chosen randomly and without specific features. All 54 patients had primary rhinoplasty using the LIS technique. Fifty patients were female (93\%) and 4 (7\%) were male (-Table $\mathbf{1}$ ). Average age was 26.4 years with minimum variation of 14 years and maximum of 62 years. Twenty-six patients were between 21 and 30 years. Only 2 (3\%) were between 51 and 70 years ( - Table 4 ).

For the development of this study, 54 patients having rhinoplasty to improve nasal tip definition through lateral intercrural suture were selected. Among the main indications for the use of this suture technique included the criteria of minor and moderate deformities, meaning domal divergence angle wider than 30 degrees, normal domal arch smaller or equal to $4 \mathrm{~mm}$ or moderately wider, larger, or equal to $4 \mathrm{~mm}$ (-Figs. 7 and 8).

Contraindications to the performance of the lateral intercrural suture included anatomic situations in which the domal divergence angle is preserved. Situations such as narrow nasal tip; asymmetric, excessive bulbosity; hyperprojected nose with excessive nasal rotation; and congenital anomalies were also among the contraindications. Patients with rounded nasal tip, bifid LLC, and weak LLC and with improper support and other conditions also required a careful evaluation.

\section{Photographic Evaluation}

Photograph study was performed with all patients with photos taken from the following views: frontal, base, submental, left and right oblique, left and right profile, at rest, and smiling. The utilized equipment was a digital NIKON B90 with AF-S NIKKOR 18-105-mm lenses and neutral background (Nikon, Inc., Japan).

For the frontal position register, the patient stood while the surgeon (observer) framed the whole face, including the ears, in the image. For the basal view, two images were captured: one with the nasal lobe alignment with the medial corner of the eyelid as parameter, and the other one with the tip of the nasal lobe aligned with the glabella. Left and right oblique visions were vertically aligned, with the nasal lobe tip meeting the pupil of the contralateral eye.

The distance for capturing the photographic images was standardized at $1 \mathrm{~m}$ between the camera lens and the nasal tip, using a fixed lens with a $100-\mathrm{mm}$ zoom to avoid distortions. The focus was placed on the nasal tip. Results were assessed by a comparison between photographs from preand postoperative periods.

Other aesthetic parameters, such as projection and rotation of the nasal tip, nasal base proportion, upper and middle thirds of the nose, were not assessed in this study. The skin of the nose tip was classified as thin, medium, and thick according to the surgeon subjective assessment. Data were stored on SINPE protocol.

Table 1 Comparison of the interdomal distances in the several periods in the complete group

\begin{tabular}{|l|l|l|l|l|}
\hline \multirow{2}{*}{ Operative phases } & $\boldsymbol{n}$ & \multicolumn{2}{|l|}{ Interdomal distance $(\mathbf{m m})$} & \multirow{2}{*}{ Mann-Whitney $\boldsymbol{p}$} \\
\cline { 2 - 4 } & & Min-max & Average \pm SD & \\
\hline Preoperative & 54 & $10-16$ & $12.3 \pm 1.6$ & 0.0000 \\
\hline Perioperative & 54 & $6-10$ & $8.1 \pm 0.9$ & \\
\hline Preoperative & 54 & $10-16$ & $12.3 \pm 1.6$ & 0.0000 \\
\hline 6-mo postoperative & 54 & $7-11$ & $9.1 \pm 1.0$ & \\
\hline Perioperative & 54 & $6-10$ & $8.1 \pm 0.9$ & 0.0003 \\
\hline 3-mo postoperative & 54 & $7-11$ & $8.8 \pm 1.1$ & \\
\hline Perioperative & 54 & $6-10$ & $8.1 \pm 0.9$ & 0.0000 \\
\hline 6-mo postoperative & 54 & $7-11$ & $9.1 \pm 1.0$ & \\
\hline
\end{tabular}

Abbreviations: Min-max, minimum and maximum values; SD, standard deviation. 


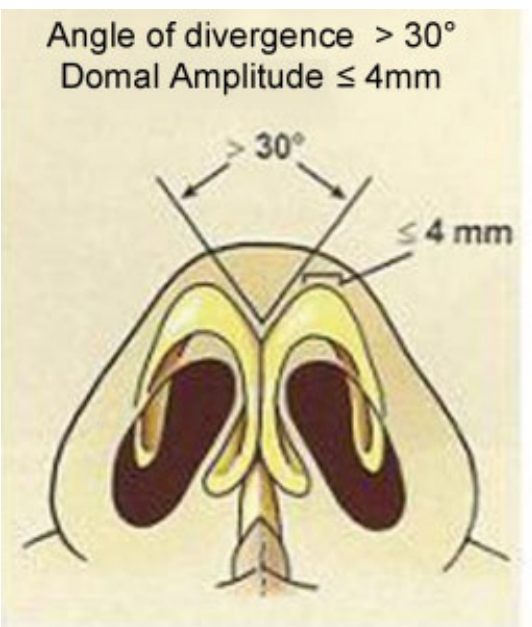

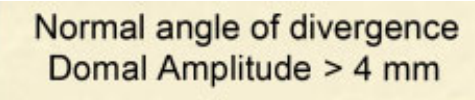

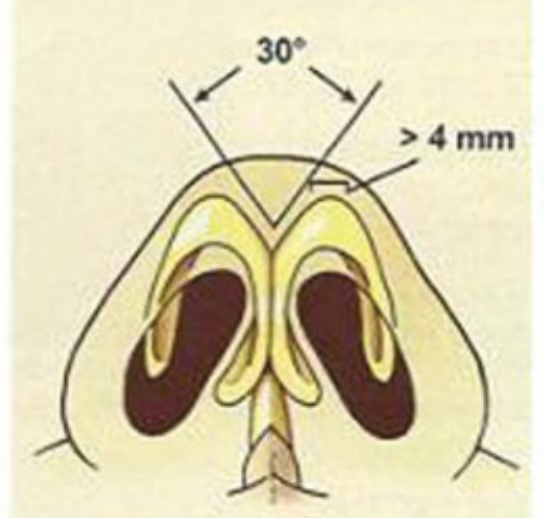

\section{Angle of divergence $>30^{\circ}$ \\ Domal Amplitude $>4 \mathrm{~mm}$}

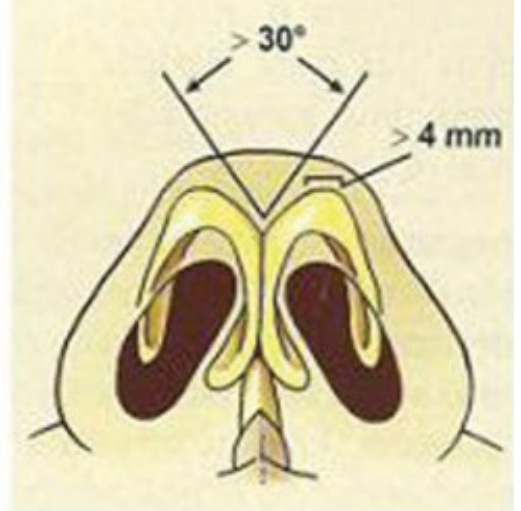

Fig. 7 Diagram indicating possible variations of nose tip types considering the variables: domal divergence angle and domal wideness according to Gunter. Source: Gunter et al. ${ }^{41}$

\section{Measurement of the Interdomal Distance}

The method used to assess the efficacy of this suture technique was the palpation of the LLCs, identifying the left and right domes of the nose and measuring the distance between them with a beam compass ranging from 0 to $20 \mathrm{~mm}$. The exact point of measurement was the midpoint between cephalic and caudal borders of the LLCs on the dome level. Those distances were measured preoperatively, perioperatively, and at 3- and 6-month intervals postoperatively.

Complications and intercurrences were registered at all postoperative appointment. Data collection was made according to the SINPE-specific protocol. Postoperative photos were taken under the same conditions described above during routine appointments on the third and sixth months after the surgery.

\section{Intercrural Suture Surgical Techniques}

The LIS technique is performed after lateral osteotomies. For better visualization of the lateral crura, the Millard thimble is used with one hook placed to coincide with the dome. (Note that the distance between the hook and the thimble is

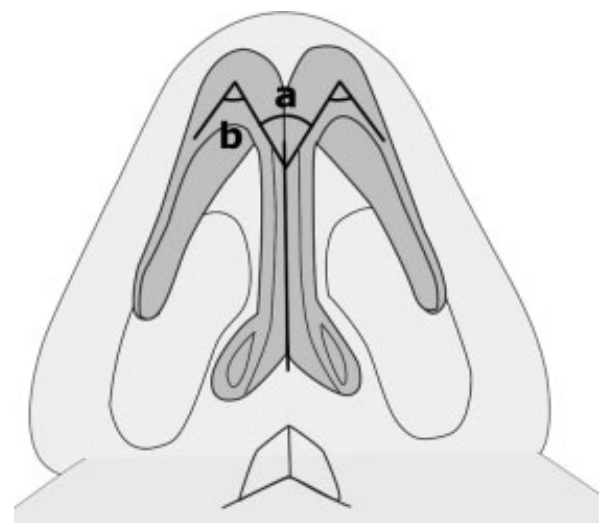

Fig. 8 (a) Domal divergence angle; (b) domal definition angle. Adapted from: Joseph and Raghu. ${ }^{42}$
$10 \mathrm{~mm}$.) Anesthetic solution of xylocaine:adrenaline $1: 100,000$ is infiltrated using an insulin needle to achieve hemostasis and hydraulic dissection separating the vestibular ski from the cartilaginous portion of the lateral crus.

With a no. 15 blade, a cutaneous incision is made, from medial to lateral, using the vibrissae caudal line as reference, and making a bipedicle flap on the medial and lateral vestibular skin using Converse angled scissors (-Fig. 9).

The removed quantity of the cephalic portion of the lateral crus of the LLC is also measured using the same beam compass. The parameter to maintain the cartilaginous arch intact, without interruption, is to preserve $5 \mathrm{~mm}$ on the medial portion and $7 \mathrm{~mm}$ on the lateral portion (-Fig. 10).

Using the same blade, these surgical steps are repeated on the contralateral area. The Le Garde maneuver is performed, which consists of the displacement of the soft tissues and connective tissues between lobular skin and LLCs (-Fig. 11).

At this moment the exact point where the LIS will be performed is marked on the lateral dome, using gentian

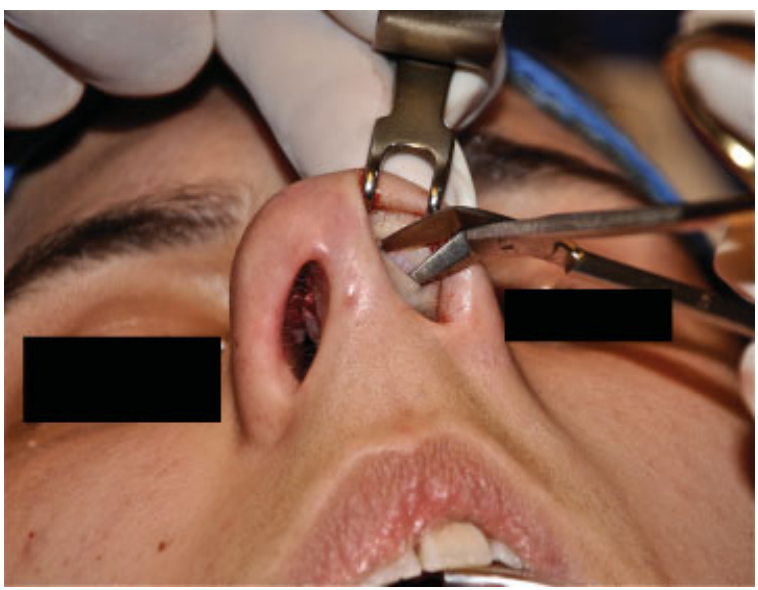

Fig. 9 Perioperative view: dissection of bipedicle flap on the medial and lateral vestibular skin using Converse angled scissors. 


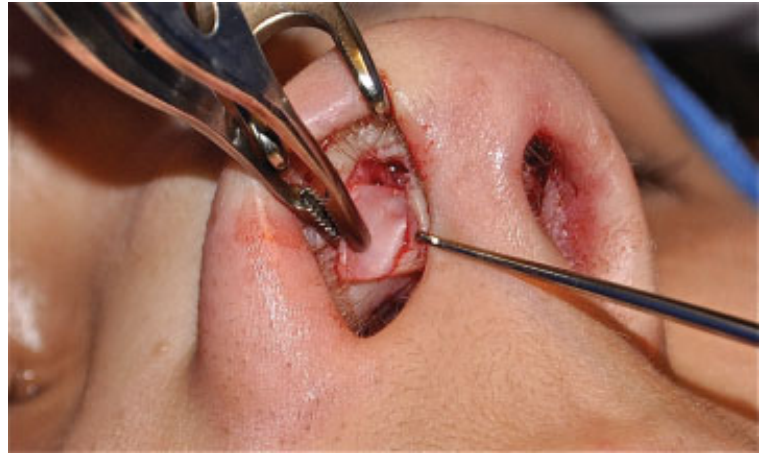

Fig. 10 Perioperative view: cephalic portion of the removed right lower lateral cartilage.

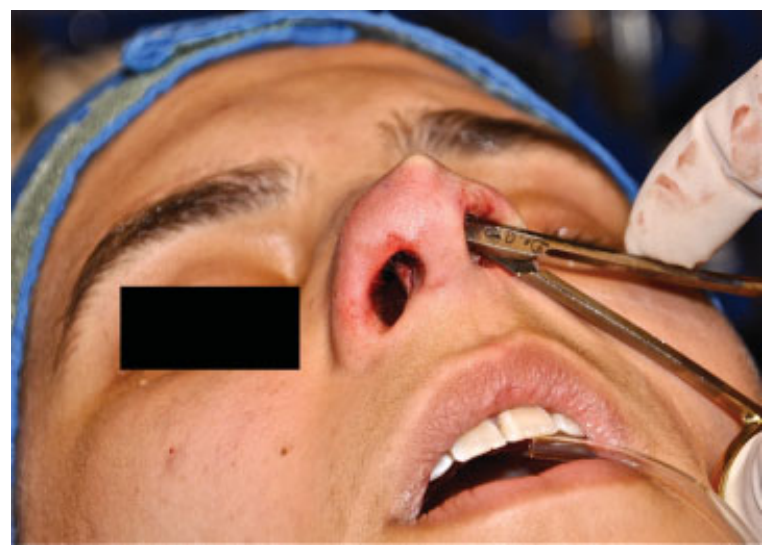

Fig. 11 Perioperative view: maneuver of disruption of the soft tissues and connective tissues between lobular skin and lower lateral cartilages.

violet. This distance varies according to the spot that supposedly can offer better anatomic results, aiming at diminishing the domal divergence angle and consequently approximating the domes. These distances can vary between 2 and $4 \mathrm{~mm}$.

Then a sharp needle with $19 \mathrm{~mm}$ of PDS 4-0 thread is inserted at cephalocaudal direction in the left LLC ( - Fig. 12).

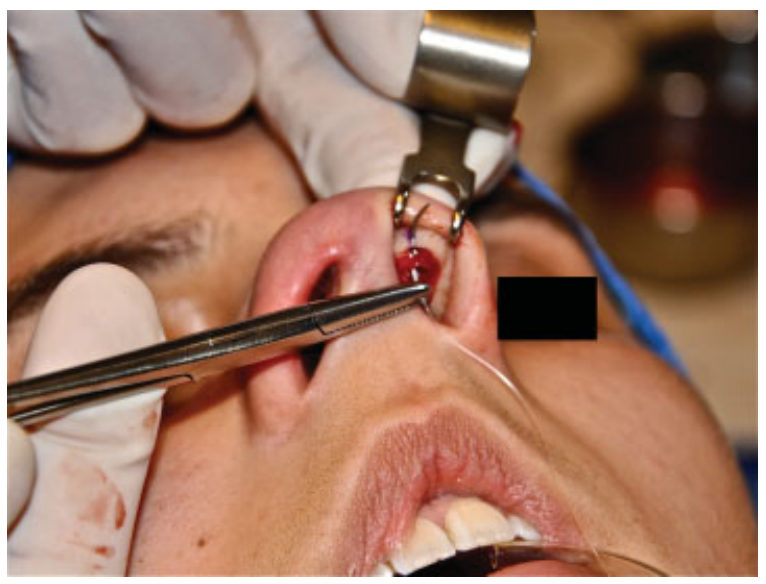

Fig. 12 Perioperative view: $3 \mathrm{~mm}$ lateral to the dome a sharp needle with a $19-\mathrm{mm}$ polydioxanone $4-0$ thread is inserted in a cephalocaudal direction in the left lower lateral cartilage.
Through the transfixion incision, the thread is transferred to the other side, placing the needle on the inverse direction, caudal-cephalic, in the right LLC at the same distance lateral to the contralateral dome (- Figs. 13 and 14). The same needle is passed through the transfixion incision to the left nasal cavity (-Fig. 15).

The knot grip is made progressively and gradually after previous simulation. It is important to mention that the knot must be centralized between the domes (interdomal space) with similar distances so that there are no asymmetries on the nasal lobe after the grip and knot are tightened ( - Fig. 16). A minimum of three knots is necessary, and a slight hypercorrection on the approximation of the domes is recommendable.

The last step includes the performance of the three septocolumellar sutures also using PDS 4-0. Once the surgery is completed, using the beam compass the new interdomal distance is registered. When compared with the preoperative distance, this distance should be smaller (-Figs. 17, 18, 19).

It is important to verify the efficacy of the dome harmonization with the aesthetic line of the nasal dorsum from an upper view (-Fig. 20).

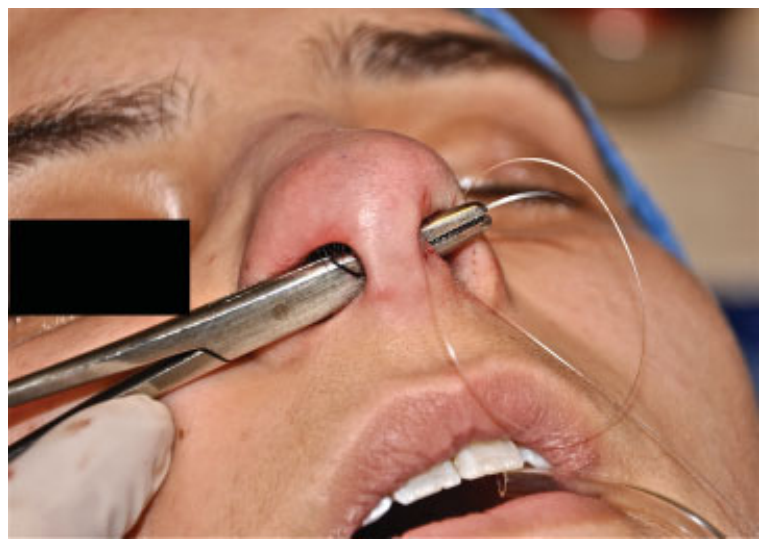

Fig. 13 Perioperative view: through the transfixion incision, the needle is passed to the right nostril.

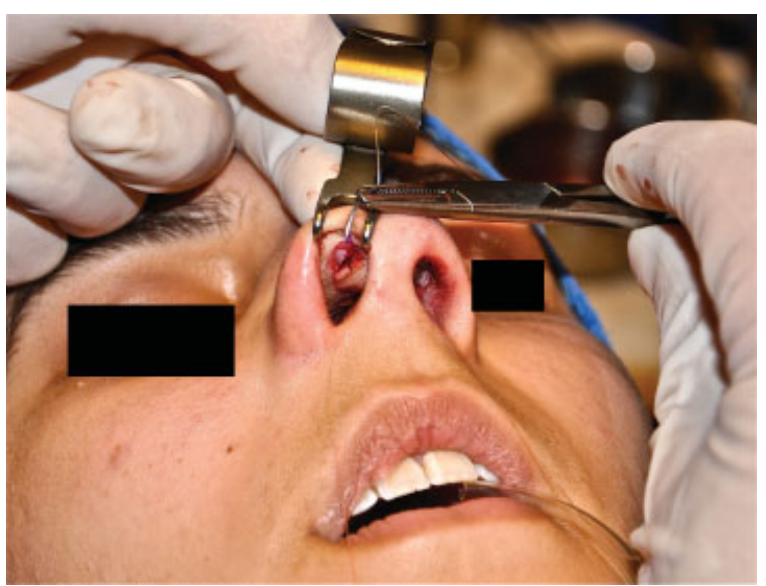

Fig. 14 Perioperative view: the maneuver is repeated on the inverse direction, caudal-cephalic, $3 \mathrm{~mm}$ lateral to the right dome. 


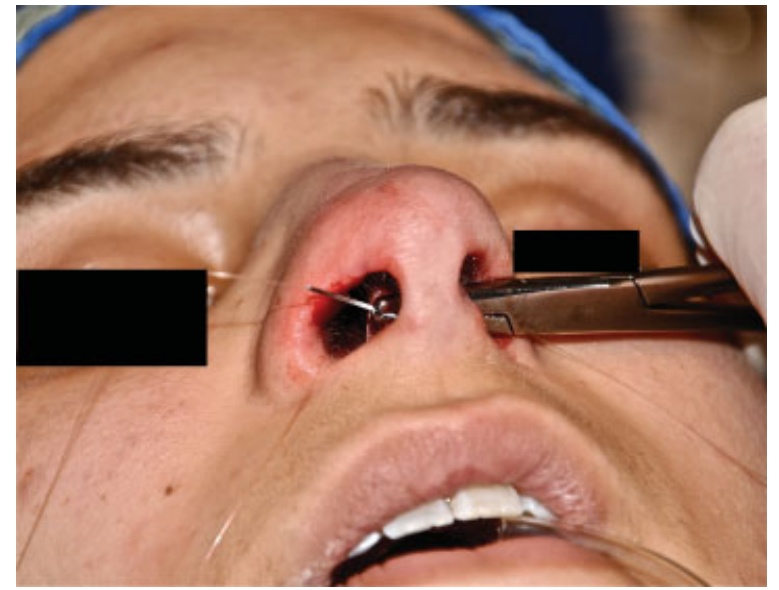

Fig. 15 Perioperative view: the same needle is passed through the transfixion incision to the left nasal cavity.

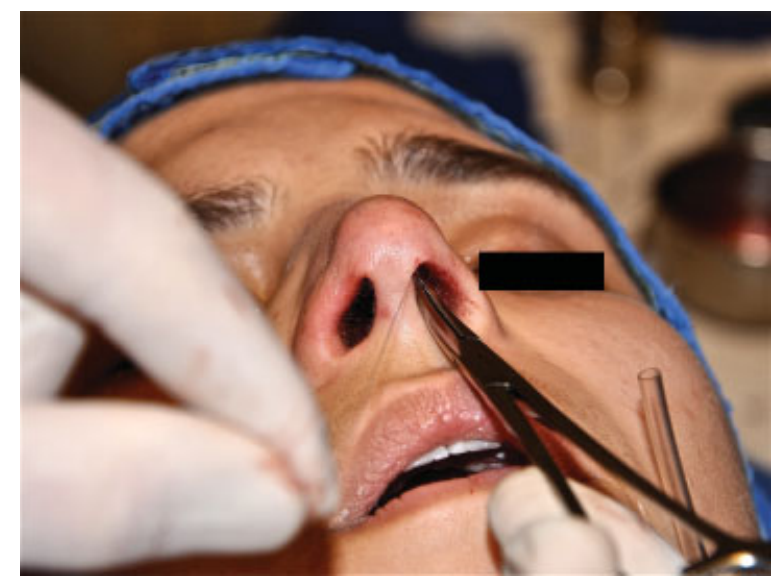

Fig. 16 Perioperative view: hemostatic clamp was used. The knot must be centralized on the interdomal space.

\section{Results}

Average age was 26.4 years, minimum 14 and maximum 62, and $44 \%$ of the sample ( 26 patients) were between 21 and 30 years. Only 2 patients (3\%) were between 51 and 70 years old.

Only one occurrence (1.9\%) was observed of an infection on the nasal tip, with no major complications. After clinical treatment using antibiotics, there were no functional or aesthetic sequelae.

The area where intercrural suture was performed was at $3 \mathrm{~mm}$ in $66.7 \%$ of the cases, at $4 \mathrm{~mm}$ on $25.9 \%$, and in only $7.4 \%$ was the suture performed at $2 \mathrm{~mm}$ lateral to the dome (-Fig. 25).

Preoperatively, the average interdomal distance was $12.3 \mathrm{~mm}$, varying between 10 and $16 \mathrm{~mm}$, as presented in -Table 1. Perioperatively, the average decreased to $8.1 \mathrm{~mm}$, with variation from 6 to $10 \mathrm{~mm}$, and at 3 months postoperatively, there was an increase on this distance reaching an average of $8.8 \mathrm{~mm}$ ( 7 to $11 \mathrm{~mm}$ ). After 6 months, the average was $9.1 \mathrm{~mm}$ (7 to $11 \mathrm{~mm}$; - Table 1).

The comparison of the interdomal distance averages between genders at different operative stages indicated an

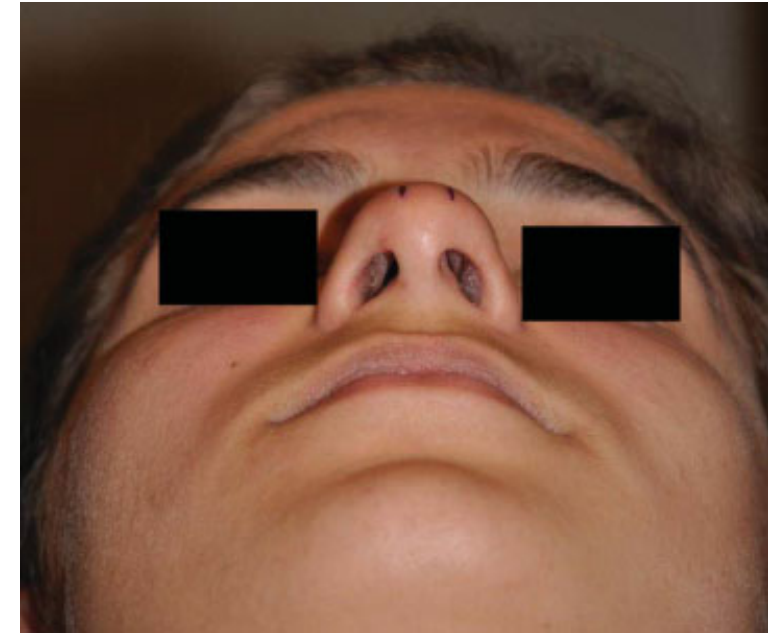

Fig. 17 Preoperative photograph (basal view).

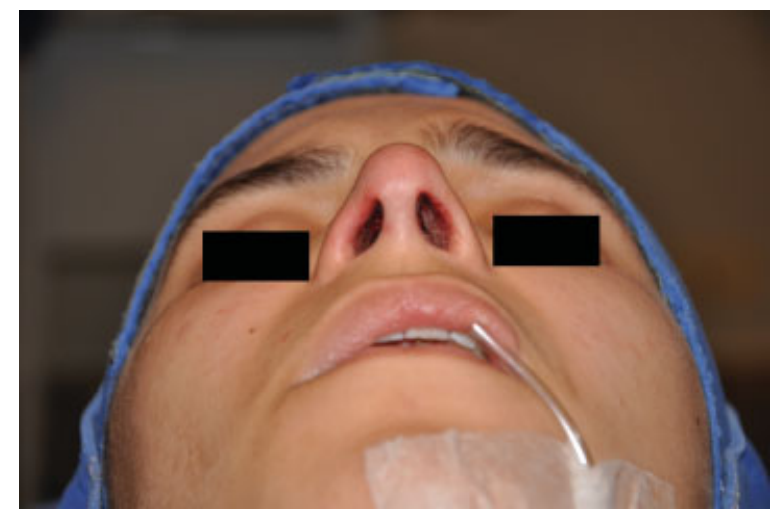

Fig. 18 Perioperative photograph with gentian violet and beam compass indicating the approximation of the $13-\mathrm{mm}$ dome reaching $8 \mathrm{~mm}$ (basal view).

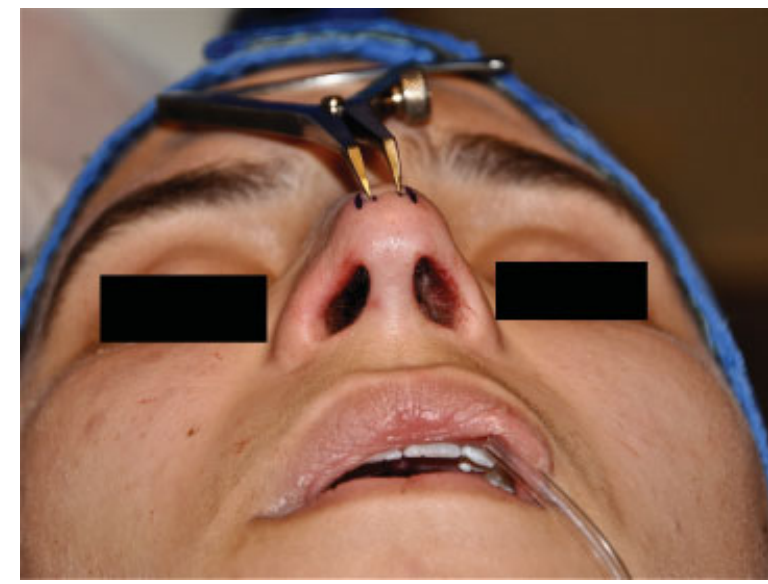

Fig. 19 Perioperative photograph indicating the decreased divergence angle between the domes, resulting on a better definition of the nasal tip. 

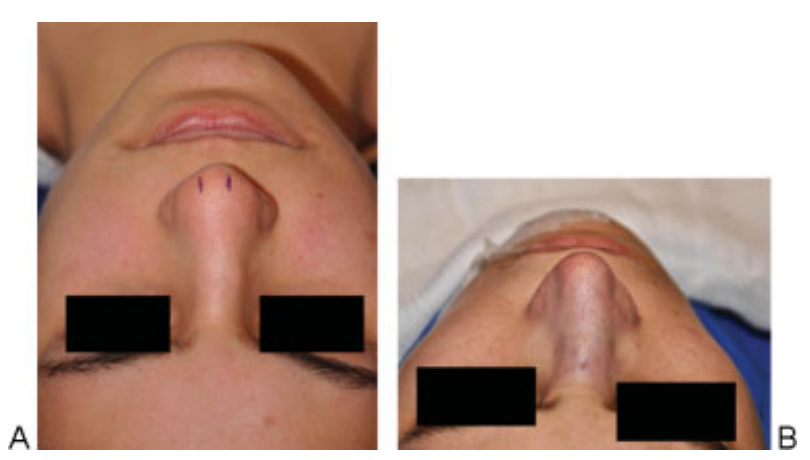

Fig. 20 Comparison of preoperative (A) and perioperative (B) photographs indicating the harmonization of the dome with the aesthetic line of the nasal dorsum.

average of $12.33 \mathrm{~mm}$ (10 to $16 \mathrm{~mm}$ ) for women and $12.5 \mathrm{~mm}$ (12 to $13 \mathrm{~mm}$ ) for men. Perioperatively, the average was $8.0 \mathrm{~mm}$ ( 6 to $10 \mathrm{~mm}$ ) and $8.5 \mathrm{~mm}$ ( 8 to $9 \mathrm{~mm}$ ), respectively, for women and men. At 3 months postoperatively, the average was $8.8 \mathrm{~mm}$ (7 to $11 \mathrm{~mm}$ ) for women and $9.0 \mathrm{~mm}$ (8 to 10 $\mathrm{mm}$ ) for men. At 6 months postoperatively, the average was $9.1 \mathrm{~mm}$ (7 to $11 \mathrm{~mm}$ ) and $9.5 \mathrm{~mm}$ (9 to $10 \mathrm{~mm}$ ) for women and men, respectively (- Table 2 ).

Regarding skin type, 27 (50\%) had medium-thickness skin. There were 22 patients with thick skin $(41 \%)$ and $5(9 \%)$ had thin skin (-Fig. 26). The average interdomal distance on patients with thin skin was $10.4 \mathrm{~mm}$ preoperatively, $7.8 \mathrm{~mm}$ perioperatively, and $8.8 \mathrm{~mm}$ at 6 months postoperatively. Therefore, there was a slight increase of $1.0 \mathrm{~mm}$ when comparing the perioperative result with the 6-month postoperative result.

For patients with medium-thickness skin, the measures of this same distance were $12.3 \mathrm{~mm}$ preoperatively, $8.1 \mathrm{~mm}$ perioperatively, and $9.4 \mathrm{~mm}$ at 6 months postoperatively. This comparison indicates an increase of $1.33 \mathrm{~mm}$ from the perioperative measurement to the final result. Patients with thick skin showed measurements of $12.7,8.0$, and $8.8 \mathrm{~mm}$, respectively, in the preoperative, perioperative, and 6-month postoperative periods. Interdomal distance on the final result increased $0.8 \mathrm{~mm}$ in relation to the perioperative result (-Tables 3 and $\mathbf{4})$.

Comparative results pre- and postoperatively and at 6-month follow-up of intercrural suture technique with cephalic resection of the LLC, at $3 \mathrm{~mm}$ lateral to the dome in patients with thin skin, showed a better definition of the nasal tip due to the reduction of the interdomal distance (-Figs. 21, 22, 23, 24).

Regarding preoperative interdomal distance of 54 patients, most (31\%) presented a $13-\mathrm{mm}$ distance; in $19 \%$ the distance was $12 \mathrm{~mm}$; and in 18\%, $11 \mathrm{~mm}$ (-Fig. 27). -Figs. 28 and 29 illustrate the interdomal distance perioperatively and at 6 months postoperatively, respectively. It was noted that $61 \%$ of the foresaid measures reached $8 \mathrm{~mm}$ perioperatively. At 6 months, $29 \%$ stayed at $9 \mathrm{~mm} ; 28 \%$ at $8 \mathrm{~mm}$; and $18 \%$ at $10 \mathrm{~mm}$. Therefore, most of the patients (85\%) presented interdomal distances measuring between 8 and $10 \mathrm{~mm}$.

\section{Discussion}

Surgical treatment of the nasal tip is one of the most important and challenging aspects of rhinoplasty. Management of the nasal tip shape involves in great part the anatomy of the LLCs and requires knowledge of aesthetic standards. The aim of this procedure is to correct the nasal tip structure by narrowing it to a harmonic definition of its contours and without interfering on the nasal function.

For many years nose tip surgery utilized aggressive techniques with wide resections or interruption of the alar arches, which may result on sequels, especially those related to sustentation and therefore to secondary deformities. Cartilage sutures, on the other hand, are a viable alternative to alterations of the nasal tip, preserving anatomic structure and improving the structural support mechanism. The first suture performed on the nose tip was described by Joseph in $1930 .{ }^{16}$ Along the two last decades, ideology among surgeons has changed very quickly, emphasizing sutures as a technique for nasal tip remodeling. The control of the tension of the suture can reduce the convexity of the dome and result in concavity of the lateral crura of the LLC. ${ }^{17}$

Table 2 Comparison of the interdomal distances between genders in the several periods

\begin{tabular}{|c|c|c|c|c|c|}
\hline \multirow[t]{2}{*}{ Operative phases } & \multirow[t]{2}{*}{ Gender } & \multirow[t]{2}{*}{$n$} & \multicolumn{2}{|c|}{ Interdomal distance (mm) } & \multirow[t]{2}{*}{ Mann-Whitney $p$} \\
\hline & & & Min-max & Average \pm SD & \\
\hline \multirow[t]{2}{*}{ Preoperative } & Feminine & 50 & $10-16$ & $12.3 \pm 1.6$ & 0.71 \\
\hline & Masculine & 4 & $12-13$ & $12.5 \pm 0.6$ & \\
\hline \multirow[t]{2}{*}{ Perioperative } & Feminine & 50 & $6-10$ & $8.0 \pm 0.9$ & 0.20 \\
\hline & Masculine & 4 & $8-9$ & $8.5 \pm 0.6$ & \\
\hline \multirow[t]{2}{*}{ 3-mo postoperative } & Feminine & 50 & $7-11$ & $8.8 \pm 1.1$ & 0.71 \\
\hline & Masculine & 4 & $8-10$ & $9.0 \pm 1.2$ & \\
\hline \multirow[t]{2}{*}{ 6-mo postoperative } & Feminine & 50 & $7-11$ & $9.1 \pm 1.1$ & 0.32 \\
\hline & Masculine & 4 & $9-10$ & $9.5 \pm 0.6$ & \\
\hline
\end{tabular}

Abbreviations: Min-max, minimum and maximum values; SD, standard deviation. 
Table 3 Comparison of the interdomal distances among skin types in the several periods

\begin{tabular}{|c|c|c|c|c|c|}
\hline \multirow[t]{2}{*}{ Operative phases } & \multirow[t]{2}{*}{ Skin types } & \multirow[t]{2}{*}{$n$} & \multicolumn{2}{|c|}{ Interdomal distance (mm) } & \multirow[t]{2}{*}{ Mann-Whitney $p$} \\
\hline & & & Min-max & Average \pm SD & \\
\hline \multirow{2}{*}{ Preoperative } & Thin & 5 & $10-11$ & $10.4 \pm 0.5$ & 0.002 \\
\hline & Medium & 27 & $10-15$ & $12.3 \pm 1.1$ & \\
\hline \multirow[t]{2}{*}{ Preoperative } & Thin & 5 & $10-11$ & $10.4 \pm 0.5$ & 0.01 \\
\hline & Thick & 22 & $10-16$ & $12.7 \pm 1.9$ & \\
\hline \multirow[t]{2}{*}{ Preoperative } & Medium & 27 & $10-15$ & $12.3 \pm 1.1$ & 0.32 \\
\hline & Thick & 22 & $10-16$ & $12.7 \pm 1.9$ & \\
\hline \multirow[t]{2}{*}{ Perioperative } & Thin & 5 & $7-9$ & $7.8 \pm 0.8$ & 0.35 \\
\hline & Medium & 27 & $6-10$ & $8.1 \pm 0.9$ & \\
\hline \multirow[t]{2}{*}{ Perioperative } & Thin & 5 & $7-9$ & $7.8 \pm 0.8$ & 0.41 \\
\hline & Thick & 22 & $6-9$ & $8.0 \pm 0.8$ & \\
\hline \multirow[t]{2}{*}{ Perioperative } & Medium & 27 & $6-10$ & $8.1 \pm 0.9$ & 0.59 \\
\hline & Thick & 22 & $6-9$ & $8.0 \pm 0.8$ & \\
\hline \multirow[t]{2}{*}{ 6-mo postoperative } & Thin & 5 & $8-9$ & $8.8 \pm 0.8$ & 0.20 \\
\hline & Medium & 27 & $7-11$ & $9.4 \pm 1.1$ & \\
\hline \multirow[t]{2}{*}{ 6-mo postoperative } & Thin & 5 & $8-9$ & $8.8 \pm 0.8$ & 1.0 \\
\hline & Thick & 22 & $8-10$ & $8.8 \pm 0.9$ & \\
\hline \multirow[t]{2}{*}{ 6-mo postoperative } & Medium & 27 & $7-11$ & $9.4 \pm 1.1$ & 0.04 \\
\hline & Thick & 22 & $8-10$ & $8.8 \pm 0.9$ & \\
\hline
\end{tabular}

Abbreviations: Min-max, minimum and maximum values; SD, standard deviation.

Note: All 54 patients were submitted to primary rhinoplasty using lateral intercrural suture technique. Fifty patients were female (93\%) and 4 (7\%) were male (see - Table 4; - Fig. 25).

Table 4 Average age in groups

\begin{tabular}{|l|l|l|l|}
\hline \multirow{2}{*}{ Groups } & \multirow{n}{*}{} & Age $(\mathrm{y})$ & Average \pm SD \\
\cline { 3 - 4 } & & Min-max & $26.4 \pm 10.4$ \\
\hline Total & 54 & $14-62$ & $26.7 \pm 10.6$ \\
\hline Female & 50 & $14-62$ & $22.5 \pm 5.3$ \\
\hline Male & 4 & $18-28$ & \\
\hline
\end{tabular}

Abbreviations: Min-max, maximum and minimum value; SD, standard deviation.

Most of those techniques aim at narrowing the tip, applying the suture precisely on a strategic spot approximating the domes and reducing its divergence angle. Sutures to create a new antihelix on otoplasty, described by Mustarde, resemble the technique of sutures in the nasal tip, on which bends and angled alterations can be performed. ${ }^{18,37}$ These techniques are safer and reversible. Sutures are used worldwide to improve nasal tip definition in endonasal rhinoplasty. ${ }^{19}$ The final results of the suture may be influenced by a number of factors, such as by the intrinsic force of the cartilage, including its thickness; by the grip of the suture; and by limitations imposed by soft tissues, ligaments, amount of subcutaneous tissue, and skin density.

The characteristics of the skin are determining factors for lateral intercrural suture efficacy. Patients with thin skin with lack of subcutaneous tissue present more expressive results. On patients with thick skin and excessive subcutaneous tissue, the LIS can present low effectiveness if compared with other techniques such as transdomal suture using semiopen rhinoplasty (delivery) or alar lateral spanning. ${ }^{20}$ Therefore, the choice of the applied technique must be made according to the skin type and the amount of subcutaneous tissue.

LIS is indicated mostly for noses with thin or medium skin and aimed at correcting deformities with an increased divergence of the domal angle, such as bifid tip, boxy tip, or round tip, with poor definition. ${ }^{21}$ Nasal tips with more complex deformities, such as hyperprojected, asymmetric, congenital deformities, or with thick skin or a disproportion between cartilage thickness and the amount of subcutaneous tissue, 


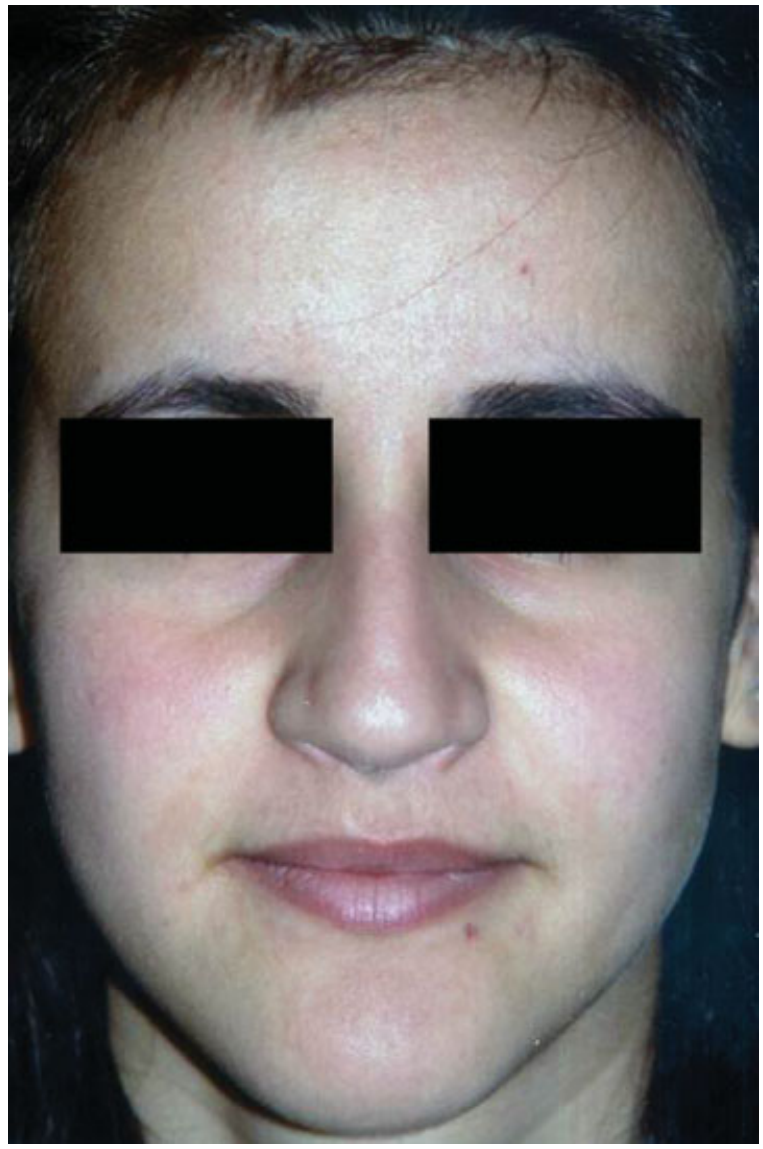

Fig. 21 Preoperative anterior view.

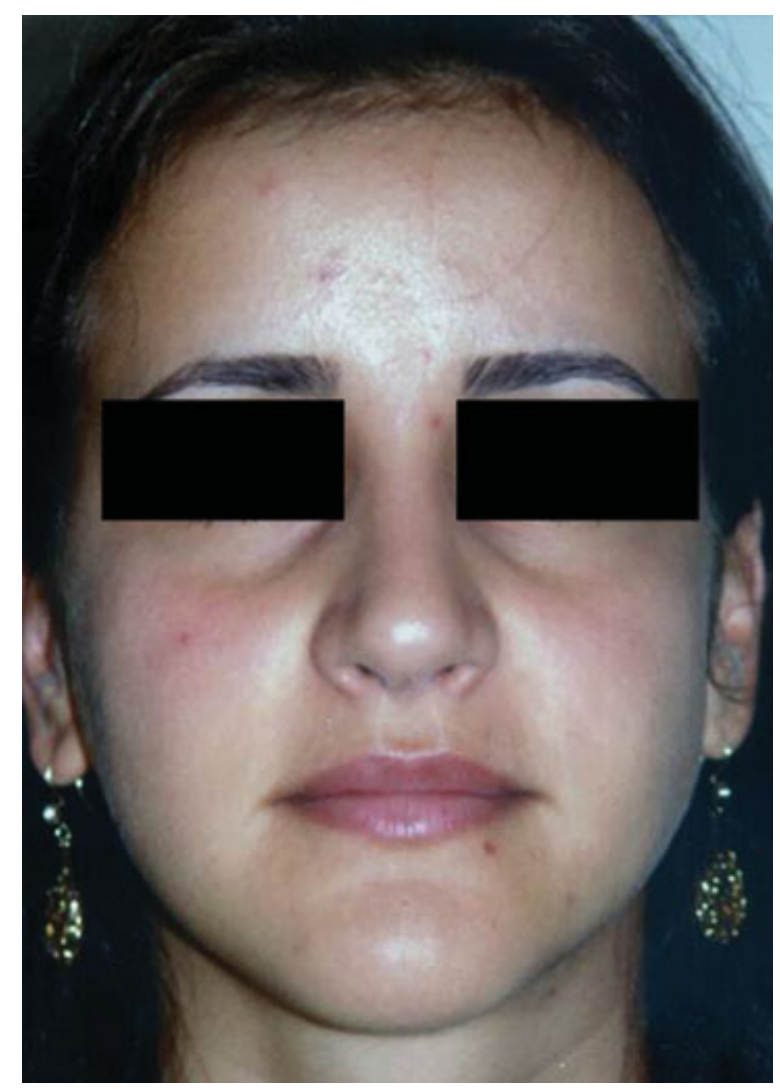

Fig. 22 Postoperative anterior view.

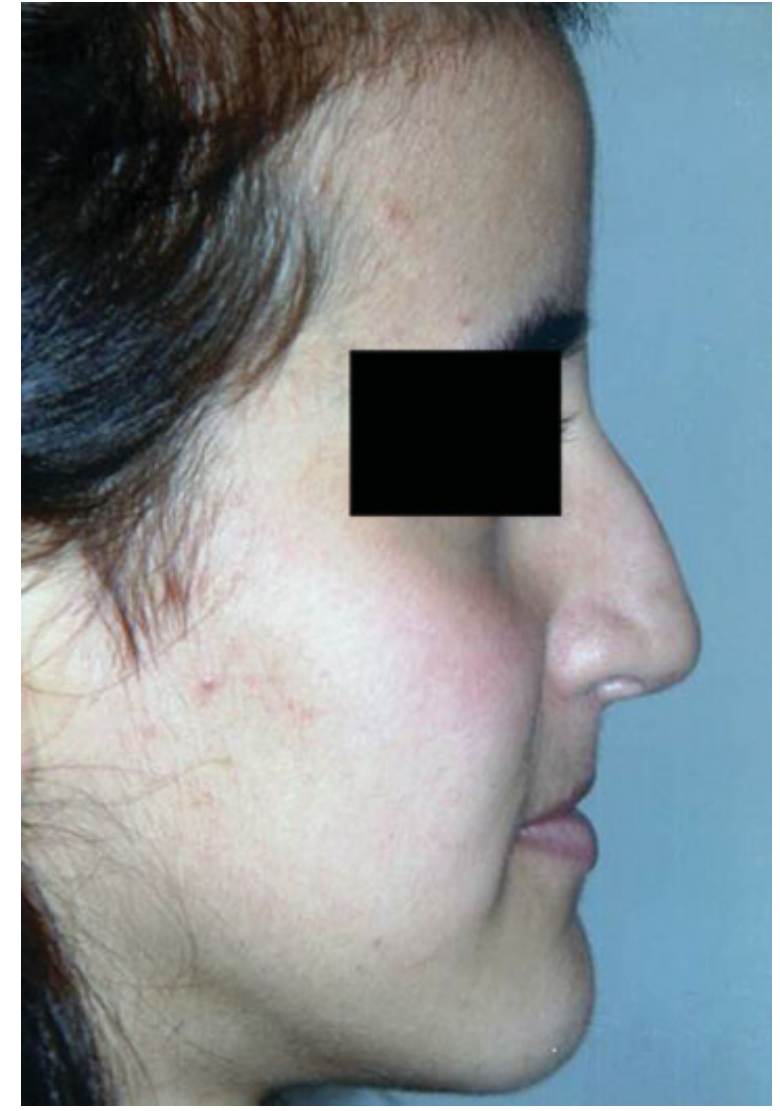

Fig. 23 View of the right profile preoperatively.

require grafts or division of the dome to achieve adequate definition and narrowing of the nasal tip. ${ }^{22}$ The authors agree that the improvement of nasal tip definition should use conservative techniques for discrete alterations on patients with thin skin and use more aggressive techniques for severe deformities. $^{23}$

LIS success depends on previous knowledge of its indications. The surgeon must consider several options of suture techniques to refine nasal tip because each nose has a unique anatomy and its harmonization may require distinct procedures. These procedures are not indicated for patients with ethnic noses, such as Asians, that present certain limitations. In these cases, strut, medial intercrural, and nasal tip grafts are used. ${ }^{24}$ There is no better technique for nasal tip definition and refinement, but different efficient methods must be customized according to the experience of the surgeon who executes them. ${ }^{25}$

It was observed that LIS sutures placed on distances more laterally to the dome, up to $4 \mathrm{~mm}$, have distinct aesthetic results. The more definition required, the more laterally the suture must be placed. Although not the object of this study, this suture may affect rotation and projection of the nasal tip.

According to Toriumi and Tardy ${ }^{18}$ and Tardy et al, ${ }^{26}$ the cartilage suture techniques have been widely applied due to the low index of complications in comparison with cartilaginous arch interruption technique. 


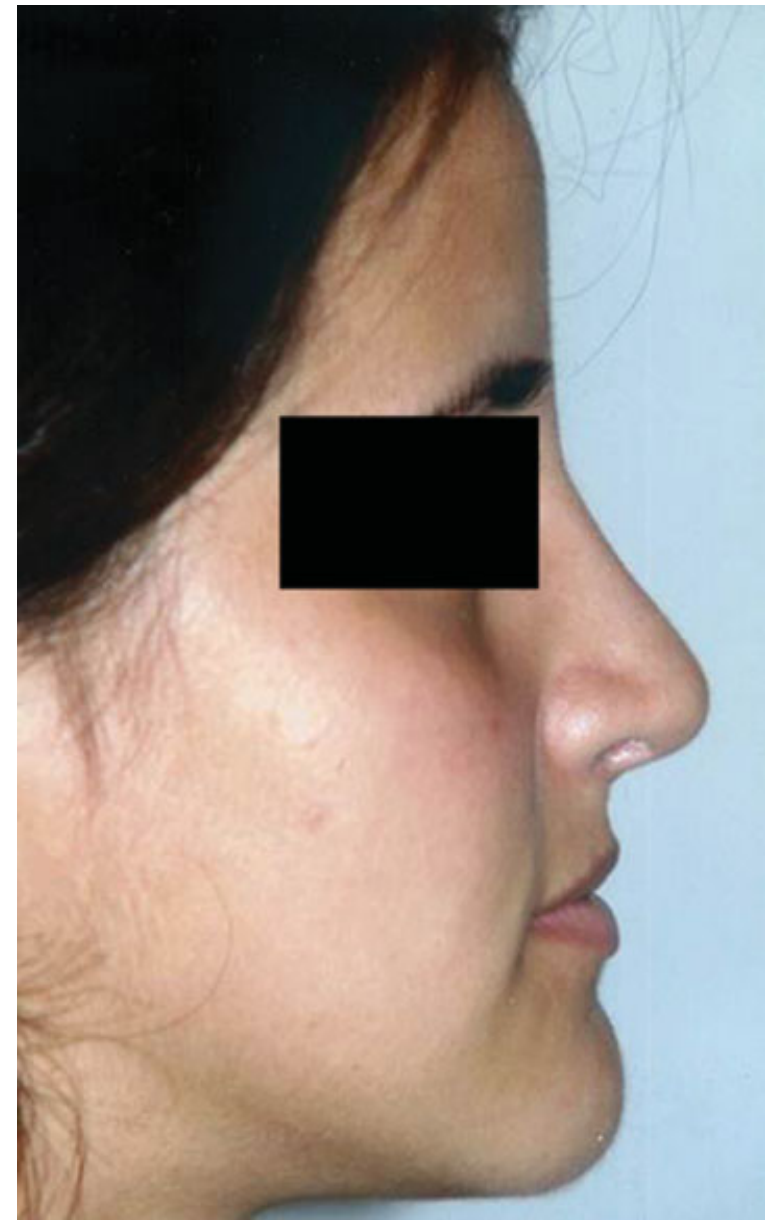

Fig. 24 View of the right profile postoperatively.

LIS can be used to efficiently build and reposition the domes. According to data in the literature, chances of extrusion of PDS 4-0 thread are very low.$^{17}$ Most complications reported are due to inadequate selection of patient or technical errors, such as asymmetric appliance of the suture. Therefore preoperative analysis is essential.

The present study observed only one case (1.9\%) of infection due to the suture, an acceptable level according to the

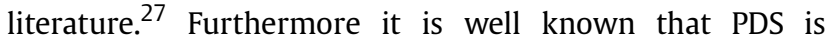

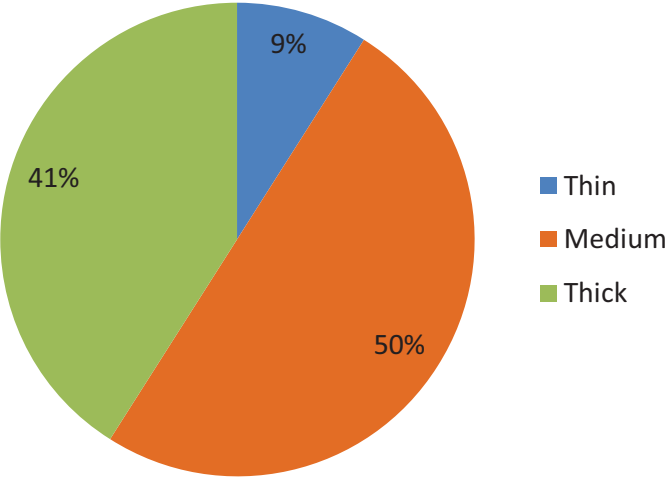

Fig. 26 Skin types of 54 patients.

completely absorbed after $\sim 180$ days. The absorption is considered slow but has the possibility of spontaneous resolution or with conservative clinic treatment using antibiotics as verified in this study. In addition to infection, the literature also reports scar retraction, abnormal tip rotation, extrusion of the suture, projection alteration, excessive narrowing of the tip, concavity of the lateral crus, supratip characterization, pinching, and valve insufficiency.

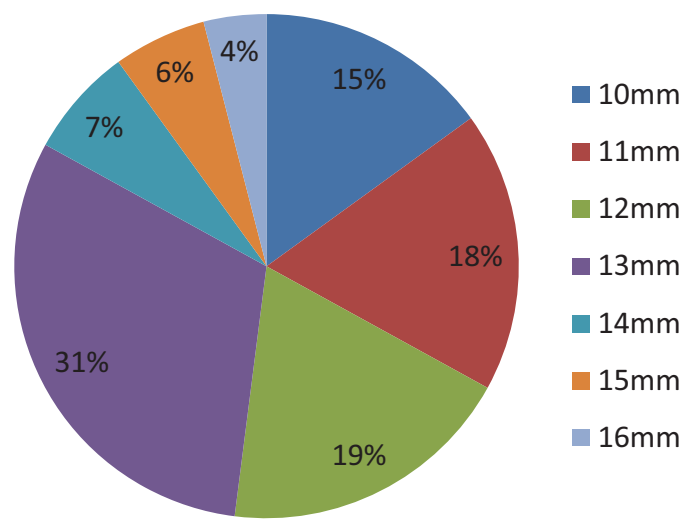

Fig. 27 Preoperative interdomal distance in 54 patients.

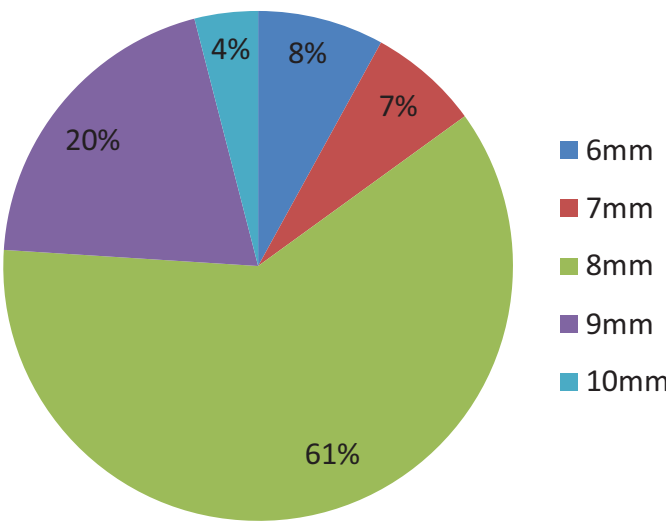

Fig. 28 Perioperative interdomal distance in 54 patients.

Fig. 25 Suture placement lateral to the dome in 54 patients. 


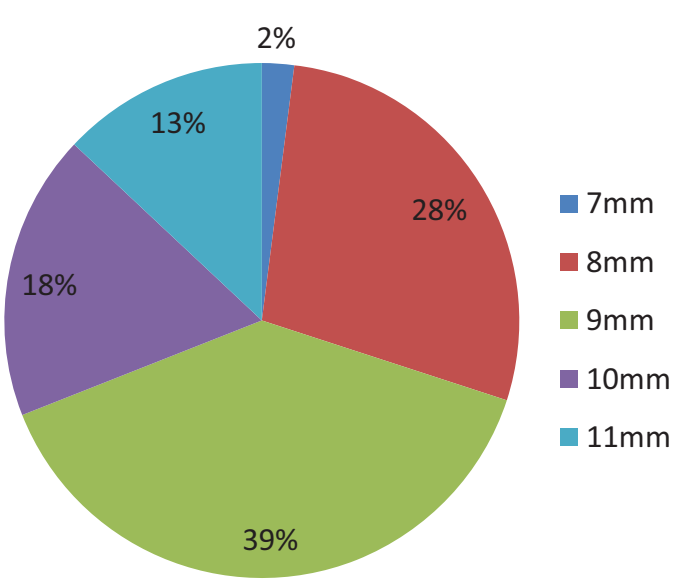

Fig. 29 Postoperative interdomal distance after 6 months in 54 patients.

Cephalic resection of the LLC is one of the most utilized techniques to reduce the width of lateral crus and was applied in all cases to obtain standardization. Access was transcartilaginous, preserving the cartilaginous arch and respecting a minimum of $5 \mathrm{~mm}$ on the medial portion, next to the dome, and $7 \mathrm{~mm}$ on the lateral portion, following the standard described by Pedroza with the "new domes" technique. ${ }^{28}$ Excessive removal may result in loss of support of the lateral crus leading to a pinching of the tip. We consider important the maintenance of these measures to preserve the support of the lateral crus, on which a sharp 1.9-cm needle was used. The grip of the knot must be gradual and always at the most natural position of the domes.

Symmetric cephalic resection of the LLC associated with LIS must have a striking and attractive aspect favoring the preservation of the triangle creating a more gracious transition on the scroll area and nasal contour, especially from a frontal view. The result would be the harmony of the aesthetic line of the nasal dorsum that must have slightly divergent curves from the supraciliary region to the nasal tip definition spots. ${ }^{29}$ All the patients had compatible symmetry regarding that aspect.

The maneuver, which consists of displacement of the soft tissues that connect the LLCs to the skin, is of great importance because it provokes secondary lobular cicatricial reaction, leading to an improvement of the harmonization of the nasal tip. It also allows the approximation and consequent reduction of the interdomal distance. The knot stays concealed at the medial line, equally distant from the dome, named the interdomal space. If this does not occur, asymmetry may become evident.

More conservative surgical approaches are necessary to correct deformities on the nasal tip. We consider the endonasal access on most cases, especially southern Brazil where there is a prevalence of Caucasian noses, requiring less reduction surgeries. Endonasal access through intercartilaginous incisions and septocolumellar transfixation is considered less destructive in comparison with semiopen and opened access. However, due to miscegenation a significant part of the population have "mestizo noses," and in these cases, there is a reduction of its structure associated with increased skin thickness and subsequent poor definition of the nasal tip, and the LIS is an option for surgical treatment.

Among the disadvantages of open rhinoplasty is the larger postoperative edema, and due to the dissection of the skin, scar retraction can distort the reconstructed osteocartilaginous structure. ${ }^{30}$ Indiscriminate fat removal must also be avoided because it increases the risks of necrosis and skeletonization of the LLC. Although not common, transcolumellar unaesthetic scar must be considered; however, advantages such as better anatomic visualization and its intercartilaginous relation are prioritized by some surgeons. We prefer endonasal rhinoplasty, but we look for results similar to those obtained through open technique. Indications for closed surgery have increased and are proportional to indications for closed and semiopen techniques. The learning curve and the comparison of results in the present study indicate the efficacy of the LIS.

The medical literature reports several techniques to structure and define the nasal tip, and they can also vary according to the preferences of each surgeon regarding suture threads. We have utilized colorless PDS 4-0, which has slow absorption characteristics, with a 19-mm sharp needle. Gruber et al had effective results utilizing colorless PDS 5-0. ${ }^{30}$ Tardy and Chemy, ${ }^{38}$ on the other hand, prefer colorless nylon $4-0$ to redirect the domes of the $L L C$, but there is no mention in their studies about this slight compensatory hypercorrection.

The possibility of extrusion of the suture thread is reduced when the knot is equidistance from the domes and covered by a subcutaneous tissue layer. Unabsorbable suture with nylon forms a minimum inflammatory reaction that devolves to a cicatricial fibrosis. After some time there is a small loss of tensional force. Similar reaction also happens with polypropylene (Prolene, Ethicon, New Brunswick, NJ), utilized by Pedroza in the new domes technique. PDS is completely absorbed after $\sim 180$ days, giving enough time for cicatrization. ${ }^{28}$ Another alternative would be PDS (3-0), which is considered more efficient regarding tensional force. The present study verified that a slight hypercorrection with interdomal approximation of $1 \mathrm{~mm}$ more than necessary may be performed, considering the structure and thickness of the LLC; the amount of subcutaneous tissue, the skin thickness, and the more lateral the dome will influence how much more tension will be necessary. Poliglecaprone (Monocryl, Ethicon, New Brunswick, NJ) would be another option, but, according to the manufacturer, its total absorption is faster, from 91 to 119 days. Thus there is no ideal thread and surgeons must be critical, particularly about absorption and loss of tensional force. Therefore more precise measurements comparing these measures preoperatively and at medium- and long-term postoperative follow-up are required.

Comparative studies with several threads must be performed and are fundamental to systematize suture techniques on the nasal tip in primary or revision rhinoplasty. Other parameters such as stabilization degree and cartilaginous support, symmetry, and objectives relative to tip projection must also be analyzed.

We prefer to measure the exact spot, lateral to the domes, then place the needle for further grip of the suture. Tebbetts 
described the lateral intercrural suture, placing the needle on the middle third of the lateral crura. ${ }^{19}$ Likewise in the present study, it is critical in that the knot must be placed at a centralized and symmetric position. The grip must be controlled to prevent excessiveness tension narrowing too much the nasal tip, increasing the concavity of the lateral crura. These sutures progressively force the lateral crura to move medially, thus the domes will be dislocated to a more caudal position with a little alar retraction.

Depending on the intrinsic forces, this suture may also result on a concavity of the alar edges, therefore requiring alar grafts. Was noticed in this study that when it is necessary to reduce the interdomal distance, the suture must be performed on the anterior portion of the dome. ${ }^{31}$ Another peculiarity that we observed was that for poorly defined tips, the LIS should be placed laterally on the dome, at $4 \mathrm{~mm}$, for example. Alar or domal arches wider than $4 \mathrm{~mm}$ or unsupported cartilages can also benefit from this technique, because in these cases lengthening of the medial crura leads to lengthening of the columella. Before gripping the knot, the surgeon must look at the nose from a tridimensional view, so that when necessary he or she can change locality in the future.

We have performed this technique using a simple suture; however, McCollough and English reported in 1985 a technique aiming at tip projection and consequent definition using a single horizontal suture connecting the four crura to the morcellized domes. ${ }^{32}$ Another technique that deserves to pointed out was published by Tardy and Chemy and consists on a previously mentioned modification technique that resects interdomal fibrous soft tissue. ${ }^{33,38}$ Gruber et al reported that the horizontal suture approximation must not be inferior to $10 \mathrm{~mm}$ approaching the domes to preserve the natural bifid aspect. ${ }^{30}$ Our study has achieved an interdomal distance of $8.1 \mathrm{~mm}$ at the perioperatively, evolving to $9.1 \mathrm{~mm}$ at 6 months postoperatively, confirming this report.

Concerning open access, Gruber et al concluded that the suture between the LLC is more precise ${ }^{30}$; however, they do not reject closed rhinoplasty technique or semiopen technique $^{34}$ with similar results. In the present study all LISs were performed using endonasal technique.

Evaluating the quantity of sutures to be performed, a single suture is considered efficient, but the necessity of a second or third complementary suture to achieve the desired effect must not be discarded. ${ }^{34} \mathrm{~A}$ parameter would be a tenuous residual convexity of the lateral crura.

Leach and Athré described 77 patients who had the technique with four sutures: intercrural medial suture, suture transdomal right, suture transdomal left, and another suture transdomal interdomal along the edge cephalic. ${ }^{35}$ They verified that these sutures affect seven variables on the nasal tip: projection, supratip, rotation, form, definition, symmetry, and bifid columella. However, only the open technique was used and follow-up was short (3 to 8 months).

They also suggest that LIS must be performed at a distance of at least 5 to $6 \mathrm{~mm}$ away from the alar edge to avoid pinching, unaesthetic scar, and preserving the aesthetic triangle described by Sheen and Sheen as well as the natural aspect of the soft triangle. ${ }^{9}$
According to Patrocínio et al on studies in which they have systematized techniques for define nasal tip, the authors prefer to perform an interdomal suture on the bulbous nose, the boxy nose, the slightly asymmetric nose, or noses with increased domal divergence angle or wider domal arch in patients with thin or medium skin. ${ }^{21}$

Our study has similar indications, but in cases where these deformities are more severe, other techniques can be associated, such as intercrural columellar strut and alar board grafts. However, patients who required complementary techniques were excluded from the present study.

Cephalic resection was performed in all cases leaving a minimum $5 \mathrm{~mm}$ from the medial portion of the LLC and $7 \mathrm{~mm}$ on its lateral portion. Patients with interdomal distance with a slight bulbosity were excluded from the study because there would be no indication for narrowing or augmentation and definition of the nasal tip.

Subtle asymmetries on the LLCs are common, and the LIS can be indicated for cases in which higher LLC can be only at one side or with unilateral widening of the domal arch and/or when the cephalic portions of the LLC have different width.

The LIS technique does not intend to substitute other techniques of nasal tip refinement that are already consecrated, such as the new domes technique described by Pedroza, where a lateralization of the domes is proposed to obtain an enlargement of the medial crus length, with transdomal and interdomal sutures using Vicryl 5-0 (Ethicon, New Brunswick, $\mathrm{NJ}){ }^{28}$ In these cases the tip usually requires more structuring, especially on negroid and mestizo noses.

LIS does not substitute for advanced techniques for nasal tip requiring extended shield graft, ${ }^{36}$ alar support graft, ${ }^{31}$ and alar contour graft necessary to correct the excessively convex lateral crura or vertically oriented. ${ }^{36}$

An advantage that must be taken into account is that this technique can be reversed if the interdomal distance gets exaggerated or if there is a reaction to the thread.

The study has verified that the profile of the patients having primary rhinoplasty aiming for a better definition of the tip indicated a prevalence of young women. Twenty-four patients (44\%) were between 21 and 30 years old. Fifty patients (94\%) were females. There was a prevalence of rhinoplasty with LIS on patients with medium-thickness skin (50\%), indicating an ethnic miscegenation in our field. Nine percent of the patients had thin skin.

Compared with the literature, our patients with thin and medium-thickness skin were more often indicated for the technique of LLC repositioning using sutures. ${ }^{30}$ We do not believe that this procedure is contraindicated for patients with thick skin; however, a more critical analysis must be made because intercrural columellar struts, grafts on the alar edge, nasal caudal septum extensors, and in some cases a graft are required. According to our statistics, $41 \%$ of the patients were classified as presenting thick skin type.

There was only one case of infection on the nasal tip, in a 15 -year-old patient at the postoperative month 3 . Conservative treatment with antibiotics was effective with improvement of inflammatory signs and no aesthetic or functional repercussion over the final result. 
It was noticed that in $67 \%$ of the LIS cases the suture was performed at $3 \mathrm{~mm}$ lateral to the dome, $26 \%$ at $4 \mathrm{~mm}$ from the domes, and $7 \%$ at $2 \mathrm{~mm}$. Thus it is possible to affirm that the sutures are efficient when placed between 2 and $4 \mathrm{~mm}$ from the dome.

It must be observed that when more projection of the nasal tip was necessary, meaning an enlargement of the medial crus of the LLC, the suture must be fixed more laterally. In this circumstance, we must be critical about the knot grip to not reduce excessively the interdomal distance, avoiding valvular insufficiency and preserving respiratory function.

Concerning the gradual grip of the LIS, we must also evaluate perioperatively the possibility of a slight increase of the supratip. There is a relation between this defect and the skin thickness, because the knot is hidden on this interdomal space and can discretely elevate the skin. ${ }^{25,30}$ That would lead to an undesired effect, a subtle "polly beak," the augment of the medium nasal third.

- Fig. 27 describes the preoperative interdomal distance in millimeters. Distances between 10 and $13 \mathrm{~mm}$ corresponded to $83 \%$ of the cases, with $31 \%$ at $13 \mathrm{~mm}, 19 \%$ at $12 \mathrm{~mm}$, and $15 \%$ at $10 \mathrm{~mm}$. Major interdomal distance was $16 \mathrm{~mm}$ in 2 patients (4\%) and the smaller was $10 \mathrm{~mm}$ in 7 patients (15\%). Our aesthetic and functional analyses indicated that the interdomal distance perioperatively can vary between 6 and $10 \mathrm{~mm}$ depending on skin type and the necessity to reduce interdomal distance in comparison to the aesthetic line of the dorsum.

The majority of the interdomal distance was around $8 \mathrm{~mm}$ (61\% of the cases). Twenty percent presented a 9-mm distance. The wider interdomal distance of $10 \mathrm{~mm}$ occurred in $4 \%$ of the patients, and the smaller one of $6 \mathrm{~mm}$ in $8 \%$ of the patients (-Fig. 27). Therefore the comparison of measures between preoperative $(12.3 \mathrm{~mm})$ and the perioperative average interdomal distance $(8.1 \mathrm{~mm})$ indicates a significant diminishing of $4.2 \mathrm{~mm}$, proving the efficacy of this technique. At 3 months postoperatively, these distances stayed between 7 and $11 \mathrm{~mm}$, with an average of $8.8 \mathrm{~mm}$, so there was an increase of $0.8 \mathrm{~mm}$ compared with the perioperative result.

At postoperative month 6 , when a more objective result can be observed, the interdomal distance stabilized at 7 to $11 \mathrm{~mm}$ with a small increase of the average to $9.1 \mathrm{~mm}$. We believe the interdomal distance at month 6 will be maintained because the average was similar to the previous measurement. The most important comparison on the evaluation of real efficacy of LIS on reducing interdomal distance would be the transition from perioperatively to 6 months postoperatively. We concluded that the average of $8.1 \mathrm{~mm}$ (perioperatively) reaches $9.1 \mathrm{~mm}$ (postoperative 6 months). Therefore, the average increase of $1.0 \mathrm{~mm}$ between these distances is acceptable. By means of statistical analysis using the Mann-Whitney test, comparative values between distances were highly significant ( $p \leq 0.05$ ). At this point, we suggest a slight hypercorrection on the LIS, around $1.0 \mathrm{~mm}$, expecting that as mentioned before, the interdomal distance will increase progressively.
Comparison of different skin thickness, the interdomal distance on thin skin patients was $7.8 \mathrm{~mm}$ (perioperatively), reaching $8.8 \mathrm{~mm}$ at 6 months postoperatively. Thus, a similar result to the general average increase of $1.0 \mathrm{~mm}$. Patients with medium-thickness skin presented a $8.1-\mathrm{mm}$ distance (perioperatively) followed by a 9.4- $\mathrm{mm}$ distance at 6 months postoperatively, indicating an augment of $1.3 \mathrm{~mm}, 0.3 \mathrm{~mm}$ more than the average. For thick-skinned patients when comparing the perioperative distance $(8 \mathrm{~mm})$ to postoperative measures $(8.8 \mathrm{~mm})$, this augmentation is $0.2 \mathrm{~mm}$, smaller than the average ( $\mathbf{- T a b l e ~} \mathbf{3}$ ).

\section{Conclusion}

The analysis indicated that the reduction of interdomal distance by the LIS technique using PDS thread diminished the domal divergence angle; approximated the domes, resulting in a better definition of the nasal tip in primary rhinoplasty; and maintained an efficient support with good reliability, low morbidity, and low complication index. Therefore this can be a complementary technique to the aesthetic harmonization of the nasal dorsum line.

It was also concluded that there was no significant difference among the obtained results regarding gender and skin types. However it would be prudent to hypercorrect that distance up to $\sim 1.0 \mathrm{~mm}$ considering the aesthetic and functional relation on noses with slight or moderate deformities.

\section{References}

1 Garcia LC. Estudo do equilíbrio dorso-ponta na rinoplastia. Simpósio Brasileiro de Contorno Facial-SBCP; 1983São Paulo, Brazil: SBCP; 1983:167-170

2 Pitanguy I. Aesthetic Plastic Surgery of Head and Body. Berlin, Germany: Springer-Verçog; 1981:257-301

3 Daniel RK, Regnault P, eds. Aesthetic Plastic Surgery. Boston, MA: Little Brown; 1993:253-255

4 Gruber RP. Suture correction of nasal tip cartilage concavities. Plast Reconstr Surg 1997;100:1616-1617

5 Toriumi DM, Checcone MA. New concepts in nasal tip contouring. Facial Plast Surg Clin North Am 2009;17:55-90, vi

6 O'Neal RM, Iremberg PH, Scheleisenger J. Surgical anatomy of the nose. In: Daniel RK, Regnault P, eds. Aesthetic Plastic Surgery. Boston, MA: Little Brown; 1993:3-39

7 Rohrich RJ, Adams WP Jr. The boxy nasal tip: classification and management based on alar cartilage suturing techniques. Plast Reconstr Surg 2001;107:1849-1863, discussion 1864-1868

8 Tardy ME. Surgical Anatomy of the Nose. New York, NY: Raven Press; 1992:36-72

9 Sheen JH, Sheen AP. Aesthetic Rhinoplasty. 2nd ed. St. Louis, MO: Quality Medical Publishing; 1997:50-92

10 Oneal RM, Beil RJ. Surgical anatomy of the nose. Clin Plast Surg 2010;37:191-211

11 Daniel RK. The nasal tip: anatomy and aesthetics. Plast Reconstr Surg 1992;89:216-224

12 Zelnik J, Gingrass RP. Anatomy of the alar cartilage. Plast Reconstr Surg 1979;64:650-653

13 Toriumi DM. New concepts in nasal tip contouring. Arch Facial Plast Surg 2006;8:156-185

14 Pitanguy I. Aesthetic Plastic Surgery of Head and Body. Berlin, Germany: Springer-Verçog; 1981:257-301 
15 Tebbetts JB. Discussion: nasal tip sutures part I: the evolution. Plast Reconstr Surg 2003;12(8):1146-1149

16 Leach JL, Athré RS. Four suture tip rhinoplasty: a powerful tool for controlling tip dynamics. Otolaryngol Head Neck Surg 2006; 135(2):227-231

17 Corrado A, Bloom JD, Becker DG. Domal stabilization suture in tip rhinoplasty. Arch Facial Plast Surg 2009;11:194-197

18 Toriumi DM, Tardy ME. Cartilage suturing techniques for correction of nasal tip deformities. Oper Tech Otolaryngol Head Neck Surg 1995;6(4):265-273

19 Tebbetts JB. Shaping and positioning the nasal tip without structural disruption: a new, systematic approach. Plast Reconstr Surg 1994;94:61-77

20 Lo S, Rowe-Jones J. Suture techniques in nasal tip sculpture: current concepts. J Laryngol Otol 2007;121:e10

21 Patrocínio LG, Patrocínio TG, Maniglia JV, Patrocínio JA. Graduated approach to refinement of the nasal lobule. Arch Facial Plast Surg 2009;11:221-229

22 Simons RL. Vertical dome division in rhinoplasty. Otolaryngol Clin North Am 1987;20:785-796

23 Song HM, Kim JS, Lee BJ, Jang YJ. Deviated nose cartilaginous dorsum correction using a dorsal L-strut cutting and suture technique. Laryngoscope 2008;118:981-986

24 Gunter JP, Friedman RM. Lateral crural strut graft: technique and clinical applications in rhinoplasty. Plast Reconstr Surg 1997;99:943952, discussion 953-955

25 Nedev PK. Cranio-caudal transdomal sutures for the nasal tip correction. Eur Arch Otorhinolaryngol 2009;266:237-242

26 Tardy ME Jr, Patt BS, Walter MA. Transdomal suture refinement of the nasal tip: long-term outcomes. Facial Plast Surg 1993;9:275-284

27 Pitanguy I. Surgical importance of a dermocartilaginous ligament in bulbous noses. Plast Reconstr Surg 1965;36:247-253

28 Pedroza FA. A 20-year review of the "new domes" technique for refining the drooping nasal tip. Arch Facial Plast Surg 2002;4:157-163
29 Friedman O, Akcam T, Cook T. Reconstructive rhinoplasty: the 3dimensional nasal tip. Arch Facial Plast Surg 2006;8:195-201

30 Gruber RP, Weintraub J, Pomerantz J. Suture techniques for the nasal tip. Aesthet Surg J 2008;28:92-100

31 Gunter JP. The merits of the open approach in rhinoplasty. Plast Reconstr Surg 1997;99:863-867

32 McCollough EG, English JL. A new twist in nasal tip surgery. An alternative to the Goldman tip for the wide or bulbous lobule. Arch Otolaryngol 1985;111:524-529

33 Tardy ME Jr, Patt BS, Walter MA. Transdomal suture refinement of the nasal tip: long-term outcomes. Facial Plast Surg 1993;9:275-284

34 Guyuron B, Behmand RA. Nasal tip sutures part II: the interplays. Plast Reconstr Surg 2003;112:1130-1145, discussion 11461149

35 Leach JL, Athré RS. Four suture tip rhinoplasty: a powerful tool for controlling tip dynamics. Otolaryngol Head Neck Surg 2006; 135:227-231

36 Rohrich RJ, Raniere J Jr, Ha RY. The alar contour graft: correction and prevention of alar rim deformities in rhinoplasty. Plast Reconstr Surg 2002;109:2495-2505, discussion 2506-2508

37 Mustarde JC. Correction of prominent ear using simple mattress suture. Br J Plast Surg 1963;16:170-176

38 Tardy ME, Chemy E. Transdomal suture refinement of the nasal tip. Facial Plast Surg 1987;4:317-326

39 Oneal RM, Bell RJ Jr, Izenberg PH, Schlesinger J. Surgical anatomy of the nose. Operat Tech Plast Reconstr Surg 2000;7(4): 158-167

40 Sheen JH, Sheen AP. Aesthetic rhinoplaty. 2nd ed. Louis: CV Nosbx; 1987:p.85

41 Gunter JP, Rohrich R, Adams WP. Dallas rinoplastia: cirurgia do nariz pelos mestres. Rio de Janeiro: Revinter; 2006

42 Joseph LL, Raghu SA. Four suture tip rhinoplasty: A powerful tool for controlling tip dynamics. Otolaryngol Head Neck Surg 2006 Aug;135(2):227-31 